\title{
Account of montane and insular speciation in some Korean megadriles (Annelida: Oligochaeta)
}

\author{
Robert J. Blakemore ${ }^{1, *}$, Seunghan Lee ${ }^{1}$ and Hong-Yul Seo ${ }^{2}$ \\ ${ }^{1}$ Biodiversity Laboratory, College of Natural Science, Hanyang Uni., Seoul 133-791, Korea \\ ${ }^{2}$ NIBR (National Institute of Biological Research), Nanji-ro 42, Seo-gu, Incheon, ROK. \\ *Correspondent: rob.blakemore@gmail.com
}

\begin{abstract}
Surveys of easily accessible or moderately remote South Korean mountains has revealed several common exotic and early species-complexes [Amynthas corticis (Kinberg, 1867) with A. diffringens (Baird, 1869), A. gracilis (Kinberg, 1867) and Metaphire californica (Kinberg, 1867)], plus an unexpected number of new native taxa. Megascolecid Metaphire muиido sp. nov. and lumbricid Eisenia muuido sp. nov. are newly described from Muuido Island, Incheon. Montane taxa are parthenogenetic Amynthas tokioensis oculo subsp. nov. that lacks male pores but is yet comparable to both Amynthas tokioensis (Beddard, 1892) and Metaphire soulensis (Kobayashi, 1938) with its possible new synonym A. chiakensis Hong \& James, 2013. Apparently unique sympatric taxa are Amynthas bangtaesan bangtaesan and A. b. confinius sup-spp. nov., Amynthas centurio sp. nov., Amynthas punicans sp. nov., Amynthas seoraksan and A. seoraksan iti subspp. nov. These are newly described and their DNA COI gene barcodes, where obtainable, are presented in a phylogram with outgroup Acanthodrilidae Microscolex dubius (Fletcher, 1887) from Lake Biwa Japan being a new exotic record for Asia.
\end{abstract}

Keywords: biodiversity, exotic species-complex, genetics, mountain fauna, soil biology

\section{INTRODUCTION}

In the spirit of Kobayashi $(1936 ; 1937 ; 1938 ; 1941)$ and Easton (1981), the current work contributes to the first author's current Japanese and Korean studies, e.g. Blakemore (2003; 2008; 2012a-f; 2013a; 2013b; 2014), and attempts to use genetic barcodes of primary types or neotypes for definitive species identity (Hebert et al., 2003; Blakemore, 2013c). Current specimens are from mountains such as Seoraksan (alt. 1,708 m) - the highest mountain in the $500 \mathrm{~km}$ Taebaek Range that edges the east coast as far down as Busan and the third highest after Hallasan and Jirisan in the south. Further collection was at nearby Bangtaesan (alt. 1,444 m) and Jeombongsan (alt. 1,424 m). Islands were also sampled, and the briefest of surveys on the isle of Muuido near Incheon unearthed two new species: a megascolecid Amynthas muuido sp. nov. and a lumbricid Eisenia muuido sp. nov., the latter comparable to endemic Eisenia koreana (Zicsi, 1972) that was surprisingly overlooked in Hong's (2000) incomplete review of Korean Lumbricidae (compare his nine or ten spp. to the twenty listed in Blake- more, 2014). The current paper complements an update of moniligastrid Drawida spp. in Blakemore et al. (2014).

\section{Materials and Methods}

Taxonomic determinations follow the classifications in Sims and Easton (1972) and Blakemore (2000; 2002; 2012f). Specimens now in $80 \%$ EtOH lodged in the NIBR facility (prefixed IV0000-) had small tissue samples taken for mtDNA COI barcode analysis (courtesy of Seunghan Lee at Hanyang University) using methods similar to Blakemore et al. (2010) with data in an Appendix. Genetic analyses and phylogram via defaults from 'MEGA 6' (www.megasoftware.net) and BLAST programs (www.blast.ncbi.nlm.nih.gov/BLAST.cgi) are compared to Genbank and ENA (www.ebi.ac.uk/ena) entries or the author's iBOL (www.boldsystems.com) 'Japanese Earthworm Types' project data (e.g. Blakemore, 2012e) as well as to ongoing Korean studies.

Taxa are arranged chronologically, compared to Fig. 1 , and then alphabetically. Discussion is confined to remarks after each species' description. 


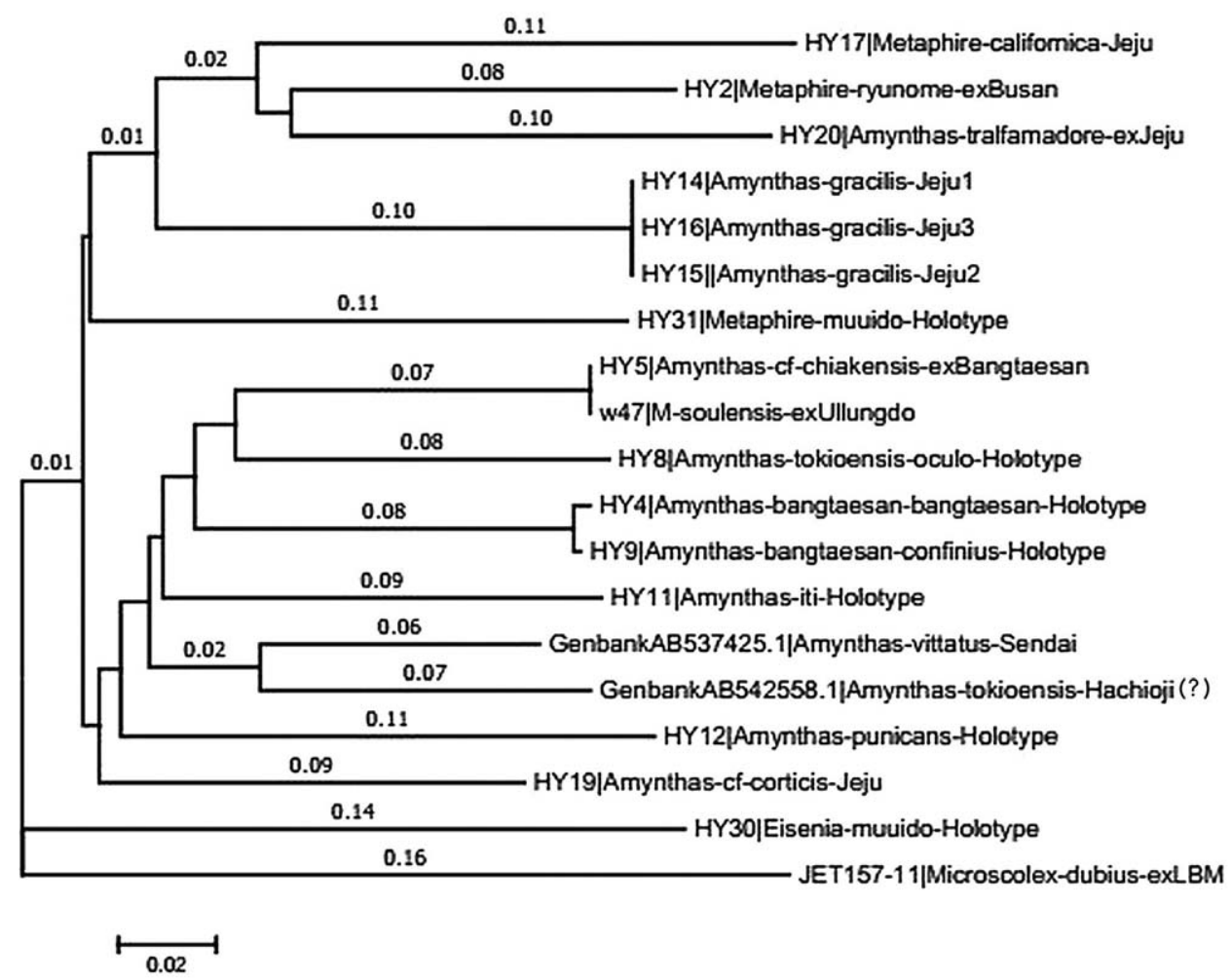

Fig. 1. MEGA6 Neighbour-Joining phylogram of DNA barcode results for evolutionary analyses using program defaults [see Tamura K., Stecher G., Peterson D., Filipski A., and Kumar S. (2013). MEGA6: Molecular Evolutionary Genetics Analysis version 6.0. Molecular Biology and Evolution 30: 2725-2729].

Abbreviations are: GMs - genital markings, lhs - left hand side; rhs - right hand side; TP - tubercula pubertates (for lumbricids); DPs - dorsal pores; neph. nephridium; "?" indicates some taxonomic uncertainty. Scale bars in all figures are $1 \mathrm{~mm}$.

\section{Genetic / Taxonomic Results and Discussion}

Annelida Lamarck, 1802

Oligochaeta Grube, 1850

Megadrilacea Benham, 1890

[Note: Some Korean websites and papers by Hong \& James incorrectly state "Phylum Annelida Linnaeus, 1758" and "Class Clitellata Linnaeus, 1740" while this grouping is actually Michaelsen, $1919 \mathrm{cf}$. defunct Class Vermes Linnaeus, 1758, also they have "Order Haplotaxida Grube, 1850" while this grouping is actually Michaelsen, 1900].

MEGASCOLECIDAE Rosa, 1891 (sensu Michalesen, 1900; Blakemore, 2000)

Amynthas Kinberg, 1867 and Metaphire Sims \& Easton, 1972 (this latter genus more for 'convenience' rather than phylogenic merit, as clearly stated by its authors).
Preliminary DNA data for taxon identification and phylogenetic relationships (Fig. 1).

1. Metaphire californica (Kinberg, 1867) (Fig. 2A).

Pheretima californica (part) Kinberg, 1867: 102 [part. excl. Megascolex indicus Horst, 1883 (=A. corticis see Easton, 1981: 49)]. [Type locality Sausolita Bay, California. Types from San Francisco in the Stockholm Museum: 160. Often misdated "1866”].

Material. IV0000261354 (DNA HY17) mature, dissected, collected from Yeomiji Botanical Gardens, Seogwipo, Jeju by RJB $5^{\text {th }}$ Sept., 2013 along with several Amynthas gracilis (Kinberg, 1867) specimens and an Amynthas cf. corticis (HY19 below).

Distribution. Widespread cosmopolitan, origin unknown; first record from Korea, also Jeju, by Blakemore (2012 et al. IV0000246440 beside Temple at Mt Sanbangsan, providing DNA sample WM15); see also Blakemore (2013a) (Saeseum Island sample providing DNA WO58). Description. Full details and distribution provided by Blakemore (2012f) "Cosmopolitan Earthworms", including specimens collected by the author from Tokyo and Nagasaki, Japan.

Remarks. Blast results for DNA sample HY17 gave 


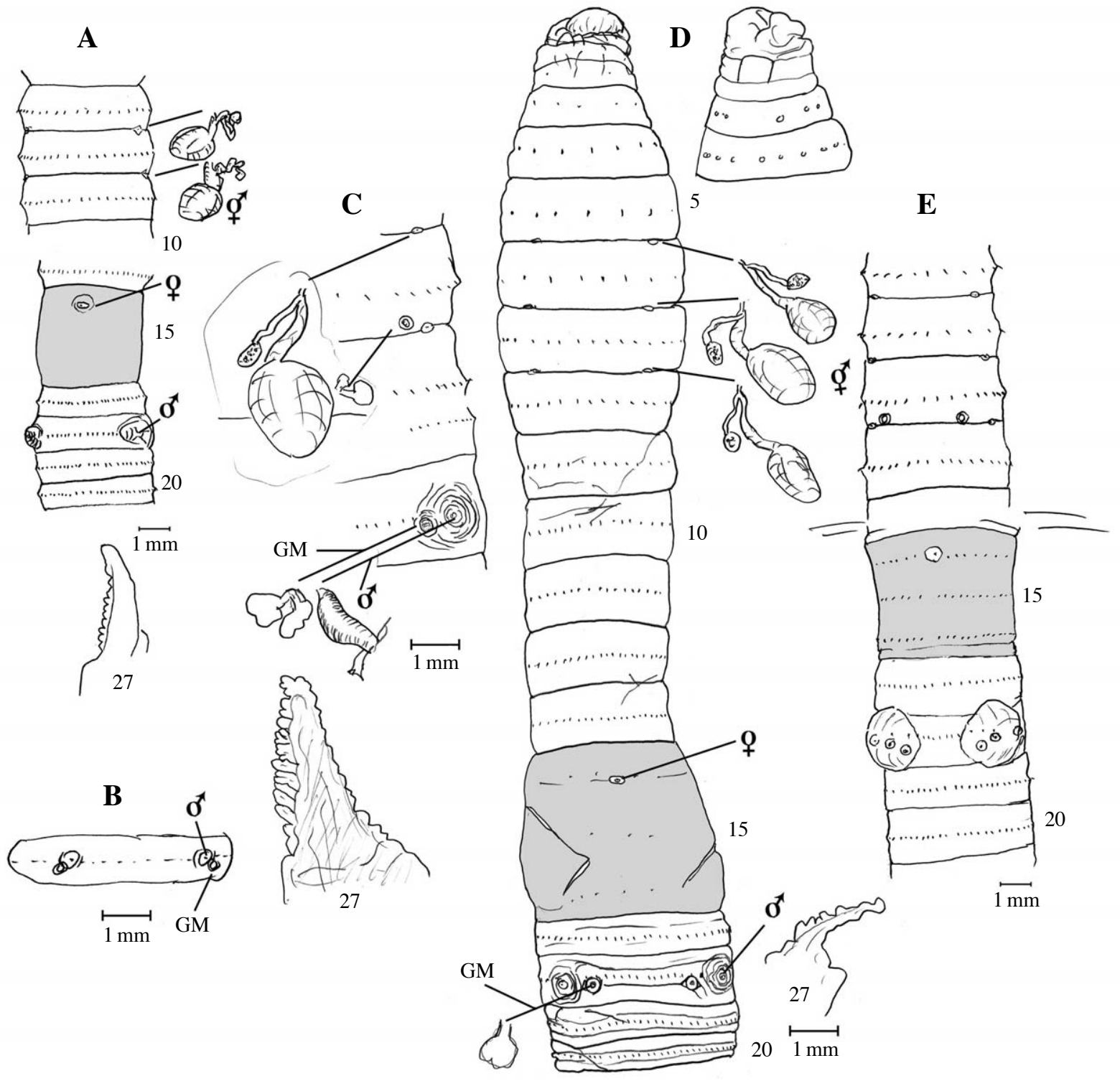

Fig. 2. 2A Metaphire californica (Kinberg, 1867) providing DNA HY17; 2B Amynthas cf. corticis (Kinberg, 1867) with GMs complying rather with A. diffringens (Baird, 1869) providing DNA HY19; 2C-E Amynthas gracilis (Kinberg, 1867) providing DNA tissue samples HY14-16, respectively.

100\% Metaphire californica (AY960810.1 from Taiwan) or 98\% "Metaphire schmardae" [EF032610 probable misidentification from China (as in Latin America!) of M. californica rather than actual Duplodicodrilus schmardae (Horst, 1883) from Japan], whereas HY17 vs. LK172-3 (M. californica from Lake Biwa, Japan $)=616 / 616(100 \%)$; plus HY17 vs. WM15 from Jeju $=617 / 617(100 \%)$ and HY17 vs. W34 (redo of WO58 from Saeseum Island, Jeju) also agrees $100 \%$. Thus my $M$. californica from Japan is confirmed the same as from Jeju, Korea.

\section{Amynthas corticis species-complex sensu Blakemore, 2003: 14}

[Consisting of A. corticis, the representative taxon, with progressive priority synonyms provided in Blakemore, 2012f].

Amynthas cf. corticis (Kinberg, 1867) (Fig. 2B).

Perichaeta corticis Kinberg, 1867: 102. [Type locality Oahu, the main Hawaii Island. Types in the Stockholm Museum: 1947, possibly immature] (part.?).

?Megascolex (Perichaeta) diffringens Baird, 1869: 40, figs. 1-3. [From Y Plas Machynlleth, Wales, UK. Type in British Museum: BMNH 1869:1:2:1 inspected by 
author (Blakemore, 2013d: fig. 9)].

Material. IV0000261360 (DNA HY19), a mature specimen from Yeomiji Botanical Gardens, Seogwipo, Jeju collected by RJB $5^{\text {th }}$ Sept., 2013. Unfortunately, IV 0000261287 , a mature specimen collected $25^{\text {th }}$ April, 2013 from Dongnae Botanic Gardens, Busan with DNA tissue sample HY3 as described by Blakemore (2013: 132) failed to yield results on second attempt and cannot be characterized further.

Distribution. Widespread cosmopolitan, origin unknown, recorded from mainland Korea, e.g., Blakemore (2013: 103, figs. 4-7), also from Jeju, e.g., by Blakemore (2012a: 26, fig. 7) [many specimens but please note: six specimens in sample IV0000251209 were mislabelled in Blakemore (2012: 26) as "IV0000251208"].

Description. Full details and distribution provided by Blakemore (2012f) "Cosmopolitan Earthworms", compared to A. corticis saeseum Blakemore, 2012a: 26, fig. 7 and to A. diffringens (Baird, 1869) by Blakemore (2013d). Remarks. The current specimen's sample HY19 appears to conform to A. corticis group-1 in the phylogram of Blakemore (2013: 101, fig. 1), e.g., $100 \%$ agreement with WM7 (A. corticis from Arataki Honey, N.Z.), "Amynthas corticis" (DQ224190.1 from Taiwan) and 100\% "Amynthas diffringens" (EF077548.1, EF077550.1 from China), also with w59 (A. corticis from Incheon) $=639$ / $639(100 \%)$ but differs slightly from w25 ("A. diffringens?" from Jeju $)=635 / 639(99 \%)$. The current dissected sample agrees superficially with $A$. diffringens type as figured by Blakemore (2013d: fig. 9) and thus may be taken as representative of this taxon.

Moreover, HY19 differs substantially from A. corticis group -2 taxa such as WM9 $(A$. corticis from Korea $)=$ $597 / 640(93 \%)$ and $\mathrm{w} 33$ (A. c. saeseum from Jeju) $=597 /$ 639 (93\%) also with JET020 and JET055 both from Japan $=597 / 639(93 \%)$ needing further clarification.

\section{Amynthas gracilis gracilis (Kinberg, 1867)}

(Fig. 2C-E).

Nitocris gracilis Kinberg, 1867: 102. [Type locality Rio de Janeiro. Types in Stockholm Museum, immatures (Sims \& Easton, 1972: 214)]; synonymy in Blakemore (2012f).

Material. IV0000261351 (DNA HY14), mature, dissected; from Seonimgyo Bridge over Cheonjeyeon Waterfalls near Botanic Gardens, Seogwipo collected by RJB $5^{\text {th }}$ Sept. 2013 found stranded on footpath at dusk; 261352 (DNA HY15) mature, ditto location but in drainage channel for rice paddy aqueduct beside footpath over the bridge; 261353 (DNA HY16), mature dissected, from Yeomiji Botanical gardens with other matures (261355358).
Distribution. Widespread cosmopolitan, origin unknown, tentatively reported from Jeju by Blakemore (2012a: 26, fig. 8A) (IV0000251208 beside Cheonji-yeon Falls providing DNA sample w63); see also A. gracilis insularum Blakemore, 2012a: 28, fig. 8B from same Jeju locality (providing DNA samples WO55, resampled as w31 and cf. w63 that both comply with "A. gracilis" from Ogasawara Islands, Japan - see Appendix).

Description. Full details and distribution provided by Blakemore (2012f) "Cosmopolitan Earthworms", compared to A. gracilis insularum Blakemore 2012a: 28, fig. 8 .

Remarks. Blast results for all three samples HY14-16 agree 100\% with Amynthas gracilis (Genbank AB5424 89.1 - mistakenly cited previously as AB542589.1 from Kagoshima on Kyushu and AB542491.1 from Yona, Okinawa; also JX177860 from Rio Grande do Sul, Brazil), whereas HY14 vs. w31 and/or W63 (Appendix) have only $98 \%$ similarity. This taxon can reasonably be accepted as the proper confirmation of A. gracilis gracilis from Jeju and Korea and conforms to a basal $A$. gracilis group shown in phylogram of Blakemore (2013a: 101, fig. 1).

\section{Metaphire hilgendorfi / Amynthas tokioensis species-complex $s$. Blakemore, 2003}

Amynthas tokioensis (Beddard, 1892) confirmed from the type by Blakemore (2010: fig. 2) with full synonymy provided in Blakemore (2012f).

Perichaeta tokioënsis Beddard, 1892b: 762. [Published December, 1892 according to Michaelsen, 1900: 272]. [From Japan (probably Tokyo as in "tokioensis"). Holotype in British Museum: BMNH: 1904.10.5.166 inspected by Sims \& Easton, 1972: 181, 191 and reinspected and refigured by RJB when on loan to YNU in October, 2004 and again in NHM, London in June, 2013; the specimen was $65 \mathrm{~mm}$ long with 67 segments but looks like a posterior amputee as the last segment has setae and is blunt rather than tapering. It is brownygrey in preservative and the clitellum is darker. The specimen had been dissected by Beddard and was slightly damaged: spermathecae in 8 were removed, only one remained in the jar that I put in a calcium vial along with a detached diverticulum, also the glands from 7 rhs seemed to have been removed, as was 18 lhs prostate].

Distribution. Japan and Korea; also reported from North America [by Gates (1954: 231) and as Metaphire levis (Goto \& Hatai, 1899) by Easton (1981: 53), sometimes incorrectly as "Metaphire levis (Horst, 1893)", and possibly as Amynthas vittatus (Goto \& Hatai, 1898)].

Remarks. Full description in Blakemore (2003; 2010; 2012f). 


\section{Metaphire soulensis (Kobayashi, 1938)}

(Fig. 3A, 3B).

Pheretima soulensis Kobayashi, 1938: 131, fig. 8. [From Keiki-dō, Keijo (now=Seoul, Kyonggi-do), South Korea. Ten specimens. Types unknown].

Pheretima shinkeiensis Kobayashi, 1938: 134, fig. 9. [A specimen from Kokai-do now in SW North Korea].

Pheretima soulensis: Kobayashi, 1941a: 51, 53; 1941b: 260 (widespread in Japan).

Metaphire soulensis: Sims \& Easton, 1972: 238 (Metaphire glandularis-group); Blakemore, 2003a: 43 (syns. Pheretima shinkeiensis Kobayashi, 1938, Pheretima aokii Ishizuka, 1999; held as species incertae sedis); 2003: 43, addenda; 2005; 2007; 2010; 2012b; 2013; 2014) (syns. shinkeiensis, aokii, dageletensis).

Pheretima aokii Ishizuka, 1999b: 36, figs. 9-18; Ishizuka et al., 2000b: 180. [From around Tokyo, including the Imperial Palace and incredibly/magically without any explanation or justification whatsoever, from Korea! Types in Tokyo].

Amynthas dageletensis Hong \& Kim 2005: 129. [From Korea].

Amynthas chiakensis Hong \& James, 2013: 76. [From Korea]. Syn. nov.? (see below).

Material. NMST-An421 two mature specimens (one sketched and dissected providing DNA sample JET028, partial result, redone as JET168 Appendix); Metaphire soulensis from Shirotaki-koen, Mishima-shi, Shizuokaken, Japan collected by RJB and Y. Hiramoto, $7^{\text {th }}$ June, 2010. Specimens also at Kuzuharagaoka Shrine, Kamakura, Japan (several specimens collected 13.vi.2004 by RJB, Amanda Reid and Yuko Hiramoto in YNU collection) that agree superfically although they lack male pores and markings, but have an extra pair of spermathecae in 5/6 (cf. the smaller A. kamakurensis). NIBRIV0000261235 M. soulensis Korea labelled "21-61" and "70", mature sketched and dissected providing DNA sample WO62 nil result, redone as w38 (contamination, Appendix) collected $18^{\text {th }}$ July, 2012 by Dr H-Y Seo.

Distribution. Korea and Japan and ?China [possibly for erstwhile yamadai synonym that was restored by Blakemore (2012b) after Easton (1981: 60) states "Japan, China, Korea", but China is probably just from Kobayashi (1939) who claimed yamadai in Japan and China]. Kobayashi (1941) had described M. soulensis as widely distributed in Japan as well as in Korea.

Diagnosis. Parthenogenetically degraded morphs 50$125 \mathrm{~mm}$ long described with adiverticulate spermathecae in 6/7/8 (sometimes lacking?), and (always?) lacking prostate glands and male pores but with genital markings as clusters of two or more papillae on 18 that have corresponding glands internally in the usual position of the male pores. These papillae, when fully formed, are at

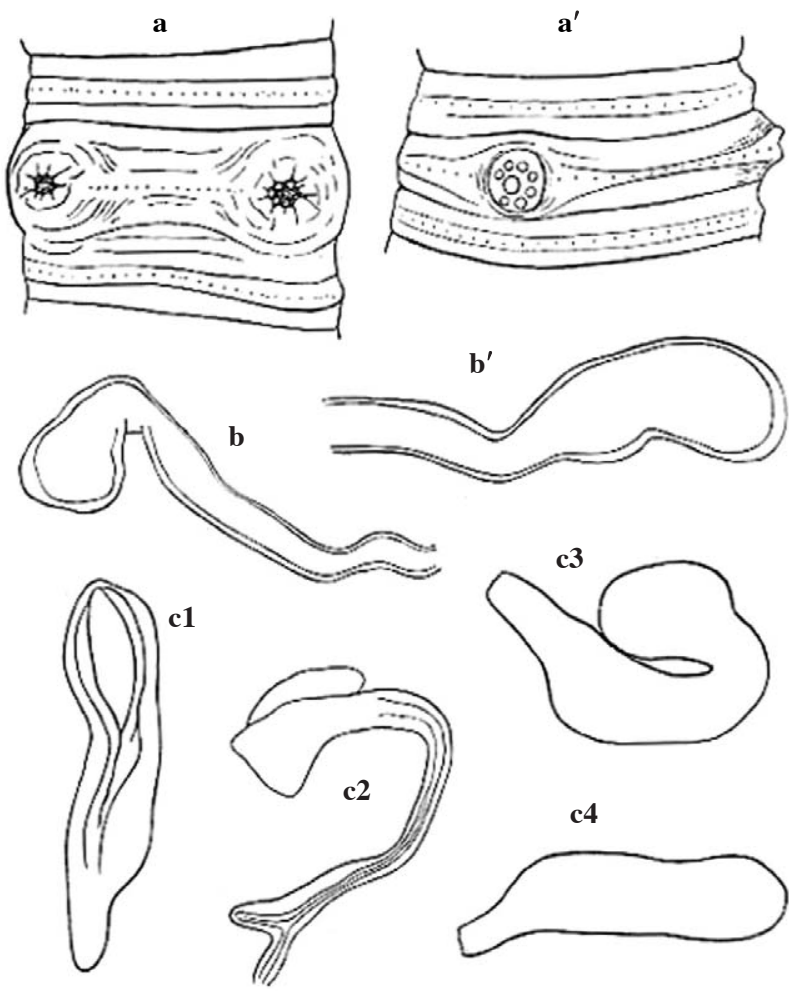

Fig. 8. Pheretima soulensis, n. sp. a \& $a^{\prime}$, ventral or ventrolateral view of XVII-XIX. Xea. 12.6 (a, Keijo-specimen, and $a^{\prime}$, Koryo-specimen). $b$ \& $b^{\prime}$, terminal ends of sperm-ducts. $X$ ea. 48.5 et-c4, spermathecac all vestirinl in size. $x$ ca. 12.6 (3-4 not eleared).

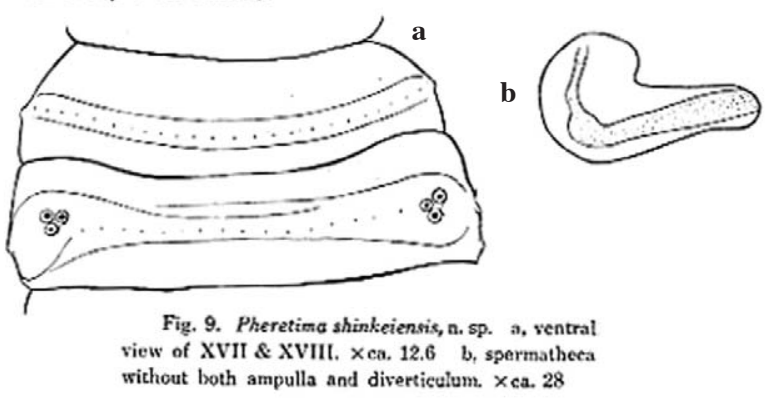

Fig. 3A. Metaphire soulensis (Kobayashi, 1938) after Kobayashi (1938, figs. 8,9) showing his Pheretima soulensis and its synonym $P$. shinkeiensis. Note that the top-right figure appears exactly the same as that of possible synonym A. chiakensis Hong \& James, 2013 (cf. Fig. 4A below).

least partly retractable into the body (hence possibly qualifying for inclusion in Metaphire see Kobayashi's original figure reproduced here). Intestinal caeca manicate in 27.

Remarks. Easton's (1981: 60) synonymy of M. soulensis (Kobayashi, 1938) in M. yamadai (Hatai, 1930), misspelt "yamardai", is not accepted here due to differences in markings around the male pores and other characters, as described on a neotype by Blakemore (2012b). Full descriptions in Blakemore (2012f). The current Korean specimen not designated a neotype because exact location 


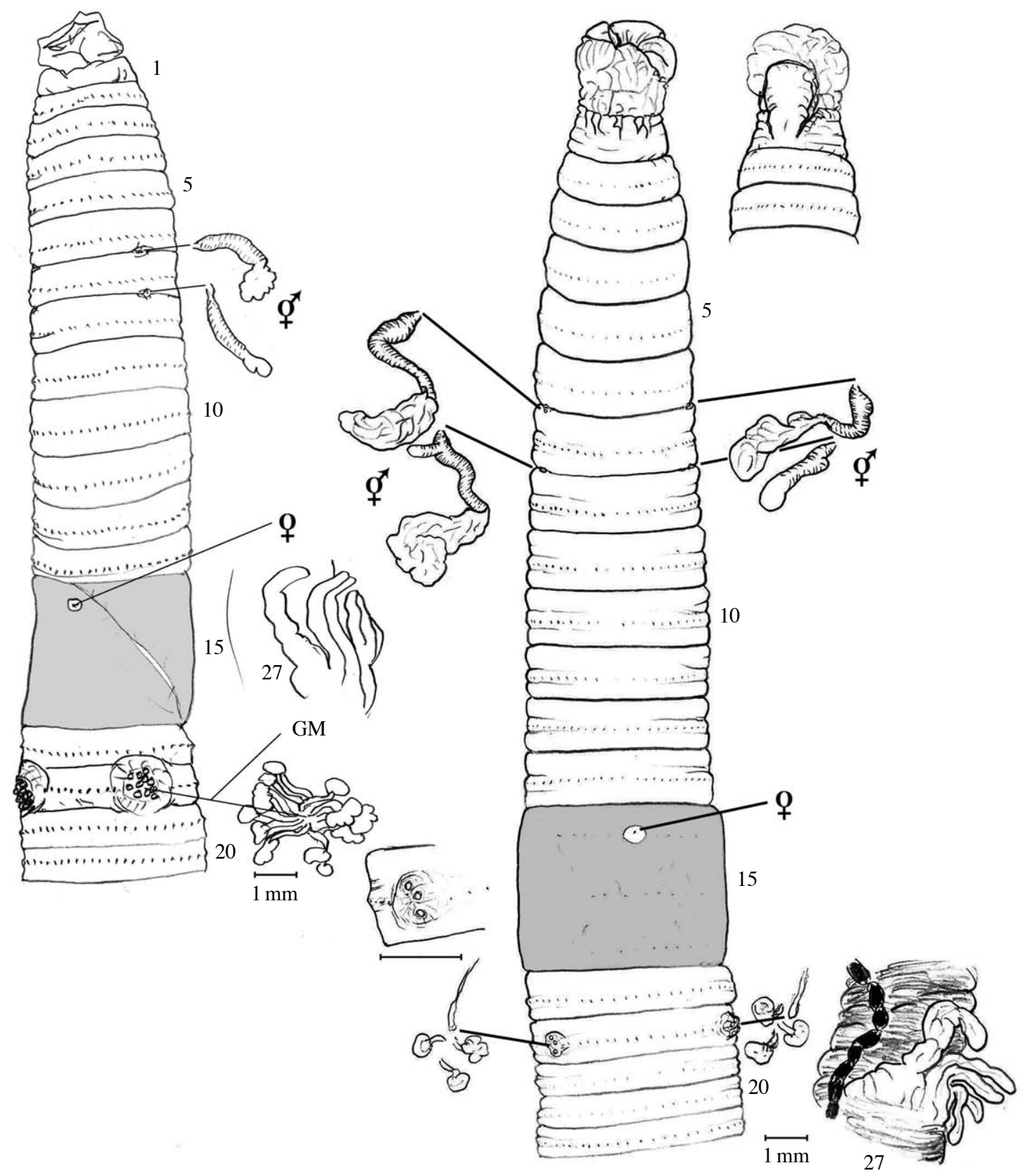

Fig. 3B. Metaphire soulensis (Kobayashi, 1938) lhs specimen is NMST An-421 from Mishima, Japan; on rhs is NIBR specimen from Korea with enlargement of its $18 \mathrm{lhs}$ male field.

not known and the DNA data was unforthcoming after two attempts.

\section{Amynthas tralfamadore Blakemore, 2012}

Material. IV0000261361 (DNA HY20) a pale mature from Jeju Town, Jeju Island by RJB $7^{\text {th }}$ Sept. 2013, found with IV0000261362 two dark matures identified as "A masatakaeltralfamadore".

Distribution. Jeju Island and introduced to NIBR facility in Incheon (type-locality).
Description. Pale specimen with extra pair GMs in $9 / 10$ otherwise complies with descriptions in Blakemore (2012d: 143, figs. 1, 7; 2013a: 35, fig. 9B-C).

Remarks. Blast result for HY20 gave 95\% "Amynthas robustus" (Genbank AB542534 - a likely misidentification from Japan), whereas HY20 vs WO2 (A. tralfamadore Holotype) Id $=619 / 621$ (99\%), i.e., tolerably the same taxon. Also HY20 vs w29 (A. tralfamadore smaller specimen from Jeju, redo of WO53) $\mathrm{Id}=618 / 621$ (99\%) and HY20 vs w30 (A. tralfamadore pale specimen from Jeju $=619 / 621(99 \%)$. Thus species confirmed from 
northern Jeju too.

\section{Metaphire ryunome Blakemore, 2012}

Material. IV0000261294 (DNA HY2) from Jungang Park, Busan by RJB $26^{\text {th }}$ April, 2013.

Distribution. Japan (Lake Biwa, Shiga-ken), Busan, Korea and "China" where it seems misidentified as "Amynthas incongruous". Thus possibly widespread of unknown origin.

Description. Provided by Blakemore (2012: 145, fig. 10; 2013: 311, fig. 4B).

Remarks. Blast results gave 99\% "Amynthas incongruous" (Genbank EF077551 - a misidentification from China). Comparing HY2 vs. M. ryunome Holotype from Hikone, Japan (Tokyo An-457, JET108) results: Id.= $653 / 653(100 \%)$, i.e., same taxon. Its wide distribution allows concession to yield to an as yet unknown species originating from Asia. Note: These latter data were not shown in Blakemore (2013b: 129, fig. 1 phylotree).

\section{Amynthas cf. chiakensis Hong \& James, 2013: 76, fig. 1A, B (Fig. 4A).}

Material. IV0000261301 (DNA sample HY5) a sub-adult

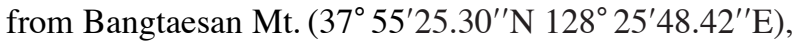
Inje, Gangwon-do, eastern South Korea, collected by Dr H.-Y. Seo, $11^{\text {th }}$ July, 2013. Found with a mature Metaphire hilgendorfi (Michaelsen, 1892) (IV0000261302), and one other specimen (IV000261300) described separately below.

Description. Length 70-82, current $60 \mathrm{~mm}$. Brown. Perichaetine. Spermathecae in 6/7/8, no genital markings nearby. Male pores superficial surrounded fore and aft by two to four papillae (three in current specimen) per side within elongate, lateral disc. Caeca manicate in 27. GM glands internally (Hong \& James, 2013: 76 note GM glands are lacking around the spermathecal pores, but this is unsurprising when there are no GMs).

Remarks. This specimen lacking anterior GMs is superficially complicit with Amynthas chiakensis (from Mt Chiak) defined by these authors as related to A. sanchongensis Hong \& James, 2001: 90 fig. 5P-T (from Mt Jiri) that has clusters of small genital markings anterior and posterior to each spermathcal pore. It was said also similar only to A. sonjaesiki Hong \& James, 2009 (from Mt Songni) that differs in having small GMs close to the spermathecal pores in 7 and 8 .

However, Blakemore (2003: 43, 2006; 2008; 2012f; 2014) suggested that $A$. sanchongensis is a synonym of A. tappensis (Ohfuchi, 1935) along with several other Japanese and Korean species that are overlooked by Hong \& James (2001; 2009; 2013). Moreover, A. sonjaesiki Hong \& James, 2009 is similarly thought a junior synonym of prior A. tokioensis (Beddard, 1892) with yet other species taking priority as per Blakemore (2003b: 43, 2006; 2008; 2010; 2012f). These issues are entirely avoided by Hong \& James (2013: 76) who merely say "Resolving the taxonomic issues associated with extensive parthenogenetic modification of sexual characters is a difficult problem in this group of earthworms, among others (cf. Blakemore 2006), and will require extensive resampling for molecular data." They yet fail to resample nor provide any molecular data to support their work, whereas in the current study COI barcodes (e.g., sample HY5 in Appendix, Fig. 1) are provided for support and comparison whenever possible.

Interestingly, the nearest BLAST match on HY5 is 621/623 (99\%) with "Metaphire soulensis" (accessions AB5426642-7 from Japan: Aomori-ken, Kanagawa-ken, Sendai City in Miyagi-ken, Hachioji near Tokyo, Kobe City and Hiroishima) and 577/579 (99\%) with > JET168 Amynthas soulensis from Japan (Appendix) also 622/623 (99\%) with DNA sample > w47 of Metaphire soulensis from Ullungdo as described by Blakemore (2013: 62, fig. 7). Current synonyms of Metaphire soulensis (Kobayashi, 1938) are Pheretima shinkeiensis Kobayashi, 1938; Pheretima aokii Ishizuka, 1999 - as per Blakemore (2003b: 43; 2012b) - plus A. dageletensis Hong \& Kim, 2005 as per Blakemore (2006; 2008; 2010; 2012b; 2013; 2014).

The question now of whether A. chiakensis Hong \& James, 2013 also belongs in synonymy of parthenogenetic M. soulensis (Kobashi, 1938) is left to its authors to determine.

\section{Amynthas tokioensis oculo Blakemore sub-sp. nov.}

(Fig. 4B).

Material. IV0000261311 (DNA sample HY8) mature from Jeombongsan Mt. $\left(38^{\circ} 01^{\prime} 16.39^{\prime \prime} \mathrm{N} 128^{\circ} 25^{\prime} 6.36^{\prime \prime}\right.$ E), boundaries of Inje and Yangyang in eastern South Korea, collected by Dr H.-Y. Seo, $25^{\text {th }}$ July, 2013. Found with four other specimens, two other Amynthas sp. described below and a Drawida jeombongsan Blakemore, 2014, plus IV0000261310 an M. hilgendorfi specimen. IV0000261308 (P) is a superficially similar mature with same details collected $11^{\text {th }}$ July, 2013 along with $\mathrm{Dra}$ wida sp. (IV0000261309).

Etymology. Latin oculo (m noun) for "black eye" referring to the look of the spermathecal pores.

Distribution. Widespread in Japan and Korea, A. tokioensis is an introduction to USA (also as synonym $M$. levis) but, in view of many misidentifications "Amynthas tokioensis species-group from central Thailand" is considered doubtful.

Description (current specimens). Length $60(\mathrm{P})$ to 85 $\mathrm{mm}$ with 90 segments $(\mathrm{H})$. Reddish-brown dorsum. Peri- 


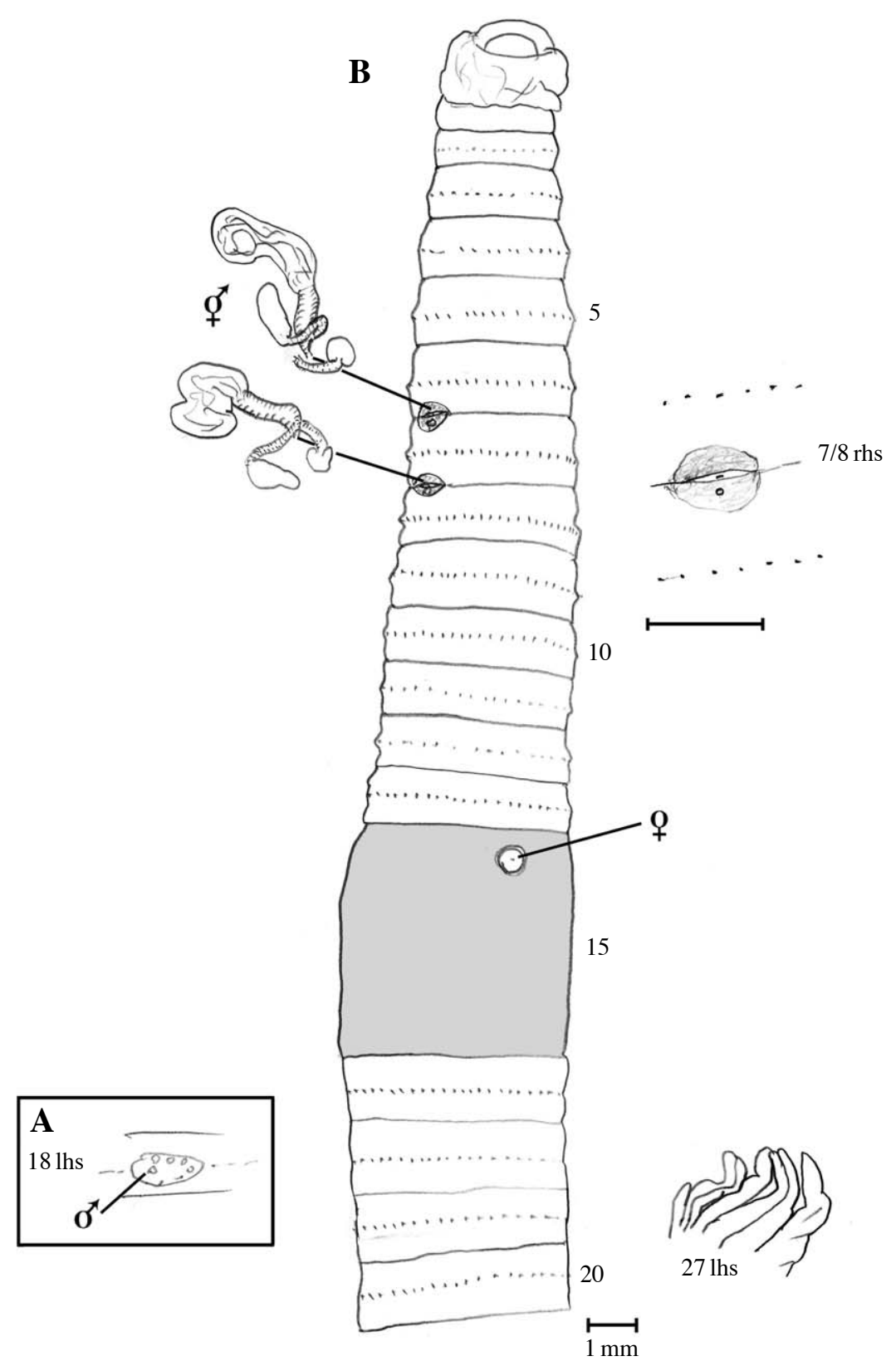

Fig. 4. 4A. Amynthas cf. chiakensis Hong \& James, 2013. Boxed sketch of male pore region (spermathecae and anterior GMs lacking). 4B. Amynthas tokioensis oculo sub-sp. nov. Holotype with enlargement of spermathecal pore 7/8 rhs (male pores and posterior GMs lacking).

chaetine with 40-50 setae. Clitellum 14-16. Spermathecae eye-like in 6/7/8 in lateral dark patches also enclosing a single GM papilla below each pore. Female pore single, central on clitellum. Male pores absent. Spermathecal ampullae deflated with clavate diverticulum on muscular duct. GM glands correspond internally to the spermathecal papillae. Seminal vesicles in 11 and 12. Last hearts in 13. Caeca manicate in 27.

Remarks. These clearly parthenogenetic specimens resemble part of the Amynthas tokioensis (Beddard, 1892) species-complex as described by Blakemore (2003; 2010; 2012f). In the latest revision by Blakemore (2014) Amynthas tokioensis (Beddard, 1892) has the following synonyms: ?schizopora, ?irregularis, levis, ?parvicystis; ?verticosa all by Goto \& Hatai, 1898/1899; ?gucheonensis Song \& Paik, 1970; jiriensis Song \& Paik, 1971; surcata and verticosa Ishizuka, 1999; ?paiki Hong in Hong, Lee \& Kim, 2001; ?yongshilensis, ?eastoni and ?boletiformis by Hong \& James, 2001; A. sonjaesiki Hong \& James, 2009 (these last nine synonyms as per 
Blakemore, 2003b: 43; 2006; 2008; 2010; 2012f; 2012g); plus Amynthas conferticurtus Hong \& James, 2009: 1241 species inquirendum and possible syn. nov.?

Amynthas conferticurtus types (IV0000120468 H \& $479 \mathrm{P}$ ) are not traceable in NIBR (pers. obs.) but it appears to be misdescribed in several key characters: e.g., the spermathecal pores are said to be on 7 and 8 (thus allegedly qualifying for an A. pomellus species group) however, they are shown in their fig. 7 to be in $6 / 7 / 8$ ! Moreover the supposed genital markings on 7 and 8 are not shown in their fig. 7 (?). Its description appears indistinguishable from their subsequent $A$. sonjaesiki Hong \& James, 2009 that was placed in synonymy of A. tokioensis by Blakemore (2010). Seemingly the A. conferticurtus name also belongs there. According to the description, it also appears to be similar to A. paiki Hong, 2001 or to A. fasciiformis Hong \& James, 2001 and both are probably in an A. tokioensis species-group, if not also synonyms. The types of the other synonym, A. sonjaesiki Hong \& James, 2009: 1243 (types IV0000120469 H $\& 480$ P) are also not traceable in NIBR (pers. obs.) but its markings resemble those claimed for A. conferticurtus. These authors need to fix these issues before they erect any further "new" names.

Regarding the current specimens, it is surprising that the nearest BLAST similarity is only $88 \%$ for Metaphire vesiculata (AB542689 from Tokyo, Okutama) or 88\% for the M. soulensis matches noted above. Fig. 1 shows separation from taxa under consideration here. It may seem precipitous to put a new name to these specimens despite the lack of a genetic match, however it will hopefully provide an unambiguous starting point for conscientious resolution of all the earliest taxa noted above progressively by any concerned workers.

Repeated searches by the senior author of the Tokyo environs for new topotypic material of Amynthas tokioensis have thus far been unsuccessful, although a record from nearby Hachioji is claimed in Genbank (accession AB542558 by Minamiya et al., 2010, unpublished). If their identification is correct, this may provide the closest match to a topotype's DNA. However, the current specimen HY8 vs. AB542558 of Hachioji=523/614 (85\%) which is far removed and questions the close identity of either or both with "Amynthas tokioensis" proper (see Fig. 1).

10. Metaphire muuido Blakemore sp. nov. (Fig. 5).

Material. IV0000261298 (DNA sample HY31) from Incheon, Muuido Island (ca. $37^{\circ} 23^{\prime} 31^{\prime \prime} \mathrm{N} 26^{\circ} 24^{\prime} 56^{\prime \prime} \mathrm{E}$ ), Hanagae beach, from treeline above northern shore, collected by RJB, $15^{\text {th }}$ Sept., 2013.

Etymology. After type-locality, noun in apposition. Description. Length $100 \mathrm{~mm}$. Segments 116. Brown

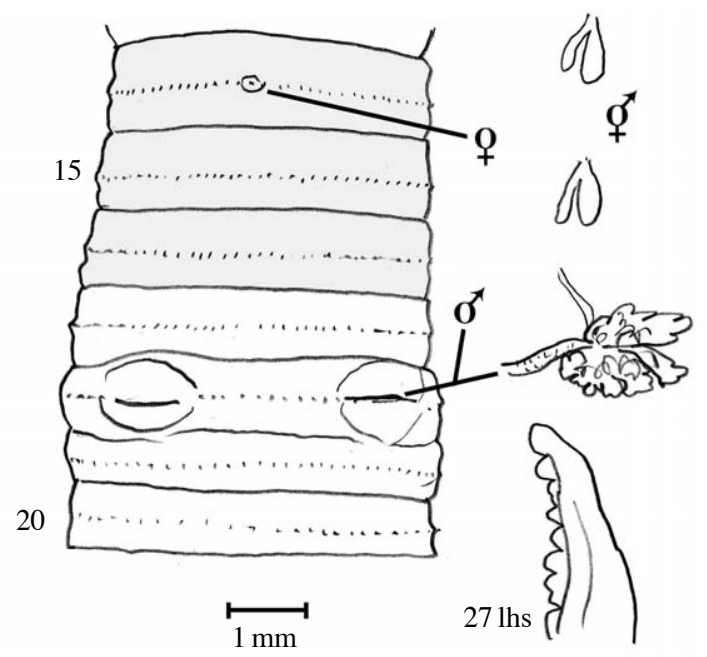

Fig. 5. Metaphire muиido sp. nov. Holotype.

dorsum, with weak clitellum 14-16. Open epilobous. First dorsal pore in 12/13. Setae ca. 74 on 12 and 15 or ca. 68 on 20. Spermathecal pores 6/7/8. Female pore mid-ventral on 14. Male pores on 18 within large trnasverse slits in round porophores filling 18 longitudinally with ca, 12 setae intervening. No trace of GMs.

Internally, large ganglia seen in segment 2. Septa 8/9/10 aborted around gizzard. Spermathecae with small, poorly developed but single clavate diverticulum and no nephridia on duct. Meroic. Seminal vesicles vestigial. Last hearts and small ovaries in 13. Prostates racemose on muscular duct but copulatory pouch not pronounced. Intestinal caeca ventrally incised from 27.

Remarks. In the rather outdated key of Sims \& Easton (1972) the non-superficial male pores of the current species keys it to the Metaphire planata-group at that time composed of six species, now doubled. The only one nearby being $M$. parvula (Ohfuchi, 56: 152) [non parvula Goto \& Hatai, 1898 see Blakemore, 2003] from the Ryukus that is smaller at $50-65 \mathrm{~mm}$, with setae on 3-8,9 long and widely spaced plus a simple rather than incised caecum; it is perhaps similar to M. decipiens (Beddard, 1912) from the Philippines that is usually (incorrectly?) placed in Pheretima darnleiensis (Fletcher, 1886). Chinese taxa added to the group are $M$. jianfengensis (Quan, 1985) that is somewhat larger at $160-250 \mathrm{~mm}$ and M. nanlingmontis plus and $M$. dadingmontis both from Guangdong by Zhang et al., 2006 having accessory glands in the spermathecal and/or prostate regions. Most other members of the group also have GMs, for example, M. planata (Gates, 1926) found in Southeast Asia has them in 7 and 8 just median to the spermathecal pores (that are actually in these segments). If the male pores were taken as superficial but in seminal grooves (unlikely as these are rarely lateral), it would qualify under Sims \& Eas- 
ton's scheme as part of an Amynthas tokioensis spp.group, this also highly outdated, especially since manicate caeca were confirmed from the type by Blakemore (2010: fig. 2) whereas the present species has simple incised caeca.

The only similar cosmopolitan species (keys from Blakemore, 2012f) is perhaps Metaphire sandvicensis (Beddard, 1896) sp. incert. sedis. from Hawaii that was initially misdescribed regarding spermathecal pores in 6/7/8, later corrected to 7/8/9 as in M. californica.

Despite its sub-adult condition, the present taxon is clearly distinguished from all other Metaphire species in Japan and Korea with spermathecae in 6/7/8 that have manicate caeca rather than simple, incised as here. Neither are the local "Amynthas" spp. with spermathecae in 6/7/8 similar, as discussed under the account of Amynthas mujuensis Hong \& Kim, 2002: 195.

Blast analysis of the DNA data in the Appendix failed to make a match closer than $87 \%$ similarity and the barcode profile now helps define this species.

\section{Amynthas bangtaesan bangtaesan Blakemore sub- sp. nov. (Fig. 6A).}

Material. IV0000261300 (DNA sample HY4) mature, rather poorly preserved, sketched and dissected from Bangtaesan Mt. $\left(37^{\circ} 55^{\prime} 25.30^{\prime \prime} \mathrm{N} 128^{\circ} 23^{\prime} 48.42^{\prime \prime} \mathrm{E}\right)$, collected by Dr H.-Y. Seo, $11^{\text {th }}$ July, 2013. Found with two specimens noted under Amynthas cf. chiakensis above.

Etymology. Named after type locality (noun in apposition).

Description. Dorsum distinctly puce but slightly translucent, ventrum paler. Length $63 \mathrm{~mm}$ with 83 segments tapering to tail. Reddish-brown dorsum. Epilobous. First dorsal pore 13/14 (i.e., at start of clitellum). Perichaetine with ca. 50-60 setae. Clitellum 14-16. Spermathecae in 6/7/8 laterally with row of six or seven GM papilla below and median to each pore. Female not found (minute). Male pores small, simple and superficial with ca. 16 setae intervening; above and below and moving ventrally from each porophore are rows of papillae, there being one row above with six or seven papillae and two below with eight to ten total. Spermathecal ampullae saccular with clavate diverticulum on thick muscular duct. Prostates racemose on straight muscular duct. GM glands correspond internally to the GM papillae. Seminal vesicles large in 11 and 12. Caeca manicate in 27.

Remarks. The nearest match for HY9 on Genbank is “Megascolecidae sp." (sic) (AB607052.1) 86\%. From the current study, HY4 vs. HY9=610/613 (99\%) which is particularly close. It is similar yet differs from Amynthas gucheonensis (Song \& Paik, 1970) in its markings which are closer to the spermathecae and only above the male pores in the latter. The type-locality for that species was Gucheon Valley on Geojedo Island and also from Mt Sopaik (or Sobaeksan?) on the mainland. The current taxon, more so than Song \& Paik's, is similar to what may reasonably be expected for a sexual form of Amynthas vittatus (Goto \& Hatai, 1898) although they compared their taxon to A. tappensis (Ohfuchi, 1935).

\section{Amynthas bangtaesan confinius Blakemore sp. nov. (Fig. 6B).}

Material. IV0000261312 (DNA sample HY9) mature, sketched and dissected from Jeombongsan $\mathrm{Mt}$, $\left(37^{\circ} 01^{\prime}\right.$ $16.39^{\prime \prime} \mathrm{N} 128^{\circ} 25^{\prime} 6.36^{\prime \prime} \mathrm{E}$ ), collected by Dr H.-Y. Seo, $25^{\text {th }}$ July, 2013; IV0000261313, superficially similar mature, same details.

Etymology. Latin 'confinius' (adj. m) implying “adjoining, contiguous or allied" for its location from neighboring Jeombongsan and similarity to nominal species.

Description. Length $62-80 \mathrm{~mm}$ with 89 segments tapering to tail. Reddish-brown dorsum. Epilobous. First dorsal pore 12/13. Perichaetine with 50-60 setae. Clitellum 14-16. Spermathecae in 6/7/8 in lateral dark patches with transverse row of six or seven GM papilla below and median to each pore. Female pore single, central on clitellum. Male pores in dark round patches that taper medially and are ringed with papillae; above each porophore is another patch of GMs each with about a dozen papillae in two rows. Spermathecal ampullae deflated with clavate diverticulum on muscular duct. Prostates racemose on U-shaped muscular duct. GM glands correspond internally to the GM papillae. Seminal vesicles large in 11 and 12. Caeca manicate in 27. Gut contains organic matter.

Remarks. The nearest match for HY9 on Genbank is only 88\% for Metaphire communissima (AB542620.1 from Japan). Morphologically distinct from the previous sub-species with regards to its dorsal pore commencement, the distribution of the GMs and possibly on the form of the prostate duct (U-shaped vs. straight), the molecular difference of HY4 vs. HY9 is slight 610/613 ( $99 \%)$ suggesting only sub-specific or ecotypic status. There may be other differences in behavior or morphology that would require further field-collection and study.

\section{Amynthas seoraksan seoraksan Blakemore sub- sp. nov. (Fig. 7A).}

Material. IV0000261306 (Holotype DNA sample HY7 - nil results) mature, sketched and dissected from Seoraksan Mt, $\left(38^{\circ} 06^{\prime} 21.34^{\prime \prime} \mathrm{N} 128^{\circ} 24^{\prime} 37.52^{\prime \prime} \mathrm{E}\right)$, collected by Dr H.-Y. Seo, $26^{\text {th }}$ July, 2013. Sample also contained an immature Amynthas sp. and an immature blue Drawida sp. (both in IN0000261307).

Etymology. After the type locality (noun in apposition). 

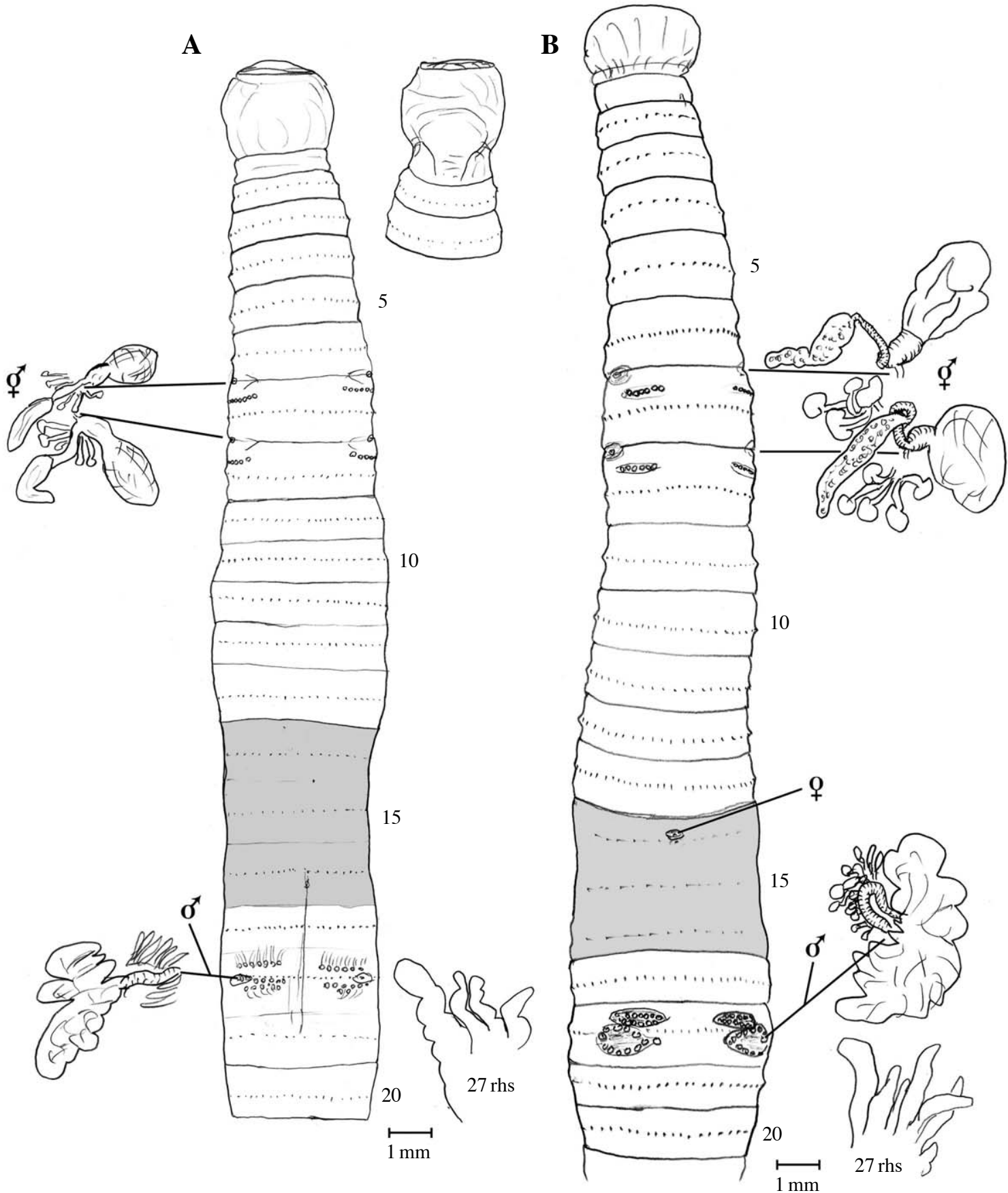

Fig. 6. 6A. Amynthas bangtaesan bangtaesan sub-sp. nov. Holotype providing DNA HY4 (female pore not found). 6B. Amynthas bangtaesan confinius sub-sp. nov. Holotype providing DNA HY9 (see Fig. 1).

Description. Length $80 \mathrm{~mm}$ with 86 segments. Bright red dorsum and pale ventrum, body somewhat transparent and tapers to tail. Epilobous. First dorsal pore 12/13. Perichaetine with ca. 40-50 setae. Clitellum 14-16. Spermathecae in 6/7/8 in lateral dark patches with tone or two GM papilla below and median to each pore. Female pore minute not found on clitellum. Male pores in setal arc within dark elongate patches containing two rows of six papillae above one or two below just medial and above each porophore; about twenty-one setae intervene between the male pores. Spermathecal ampullae saccular with clavate diverticulum on bulbous duct with GM glands nearby. Prostates racemose on bent muscular duct with glands correspond internally to the GMs. Seminal 


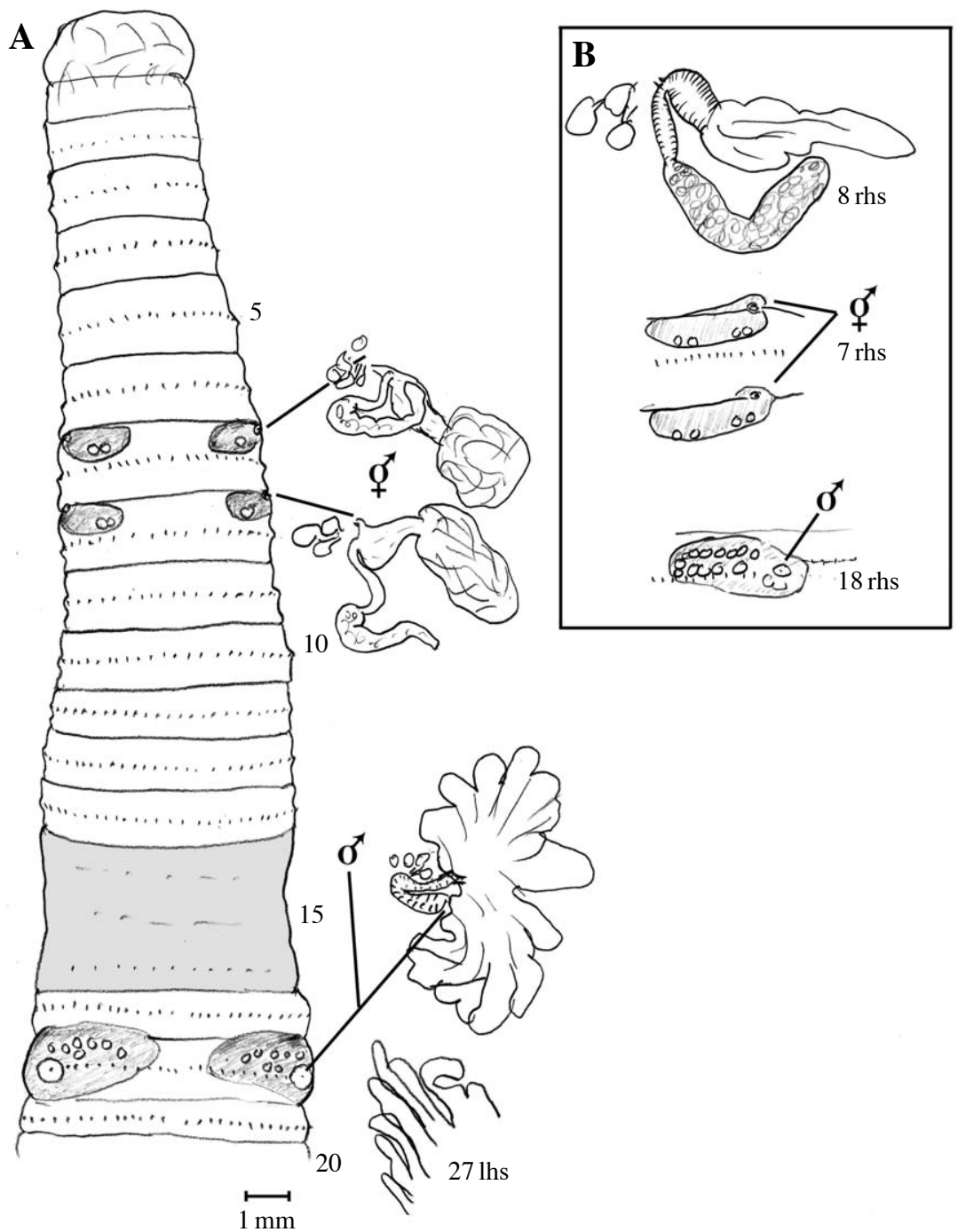

Fig. 7. 7A. Amynthas seoraksan seoraksan sub-sp. nov. Holotype. 7B. Amynthas seoraksan iti sub-sp. nov. Holotype (DNA HY11 in Fig. 1).

vesicles large in 11 and 12. Caeca manicate in 27.

Remarks. As with A. bangtaesan described above, the current species is somewhat similar to Amynthas gucheonensis Song \& Paik, 1970 from Gu-cheon Valley on Geoje Island (type locality) and Mt Sopaik on the mainland, relatively distant locations possibly accounting for supposed variability actually due to the combination of different taxa. Their species was described as similar to A. tappensis - both having GMs in spermathecal and male fields but differing in body length and spermathecal shape. Nevertheless, type descriptions differ in many small factors, mostly noticeably by lacking the bulbous spermathecal diverticula which also differentiates from A. s. iti. Unfortunately two attempts to extract and sequence DNA failed to yield genetic results, thus fresh material is required.
14. Amynthas seoraksan iti Blakemore sub-sp. nov. (Fig. 7B).

Material. IV0000261316 (Holotype DNA sample HY11) mature, sketched and dissected from Jeombongsan Mt, $\left(37^{\circ} 01^{\prime} 16.39^{\prime \prime} \mathrm{N} 128^{\circ} 25^{\prime} 6.36^{\prime \prime} \mathrm{E}\right)$, collected by Dr H.-Y. Seo, 25 ${ }^{\text {th }}$ July, 2013; IV0000261317 (Paratype), superficially similar mature, same details; plus sample contained a mature $M$. hilgendorfi specimen IV0000261315 with everted male pores (confirming its correct allocation to genus Metaphire). Other sample batch with same details contains three specimens (IV0000216318-20) that agree superficially except they may have three instead of two papillae in 7 and 8 , the longest is $120 \mathrm{~mm}$; two other samples are IV0000216321 and sympatric specimen described successively below.

Etymology. Derived from Morse code for its spermath- 
ecal markings: “.. - .."=ITI.

Description. Length $111 \mathrm{~mm}$ (both H \& P). Reddish iridescent dorsum body tapers to tail. Epilobous. First dorsal pore 12/13. Perichaetine with 50-60 setae. Clitellum 14-16. Spermathecae in 6/7/8 just above intersegment in lateral dark patches with transverse row of two sets of two GM papilla below and median to each pore (sets of two and three in some specimens). Female pore single, central on clitellum. Male pores just behind setal line in dark elongate patches two rows of about six papillae above and medial to each porophore. Spermathecal ampullae deflated with clavate diverticulum on muscular duct and GM glands nearby. Prostates racemose on muscular duct. GM glands correspond internally to the male GM papillae. Seminal vesicles large in 11 and 12. Caeca manicate in 27.

Remarks. The nearest Blast result for HY11 is also just 87\% for Metaphire communissima (Genbank AB542623.1 from Hyogo-ken, Japan), and HY11 vs. HY9 $=568 / 653$ $(87 \%)$.

Distinctive characters of $A$. iti are the markings that also resemble nesting birds (see figure) and the slightly off-line position of the spermathecal and male pores. DNA data helps confirm this identity amongst taxa tested.

\section{Amynthas punicans Blakemore sp. nov. (Fig. 8).}

Material. IV0000261322 (Holotype DNA sample HY12) mature, sketched and dissected from Jeombongsan Mt, $\left(37^{\circ} 01^{\prime} 16.39^{\prime \prime} \mathrm{N} 128^{\circ} 25^{\prime} 6.36^{\prime \prime} \mathrm{E}\right)$, collected by Dr H.-Y. Seo, $25^{\text {th }}$ July, 2013. Five other samples noted above (IV0000216318-21).

Etymology. Latin punicans (adj.) meaning "blushing" or "reddish" referring to the colour.

Description. Length $69 \mathrm{~mm}$ with 100 segments $(\mathrm{H})$. Brightest red iridescent dorsum, body tapers. Epilobous open. First dorsal pore 12/13. Perichaetine with ca. 50 setae. Clitellum weak 14-16. Spermathecae in 5/6/7 lateral. Female pore single on 14. Male pores on small hemicircular porophores equatorially with about a dozen setae intervening and medial to each porophore a small GM disc.

Septa 8/9/10 are aborted. Spermathecal ampullae smallish sacs on muscular duct with long zig-zagging, inseminated spermathecal diverticula. Testis $10 \& 11$; seminal vesicles large in $11 \& 12$. Ovaries small and not determined. Last heart in 13. Prostates racemose on circular muscular duct. GM glands not noted. Oesophagus dilated in 12-13 with intestine in 15 . Caeca single with indented trailing edge in 27. Gut contains organic soil.

Remarks. The nearest Blast result for HY12 is just $87 \%$ for Amynthas tappensis (AB542548.1 from Osaka, Japan). Distinctive characters of A. pumitans are the spermath-

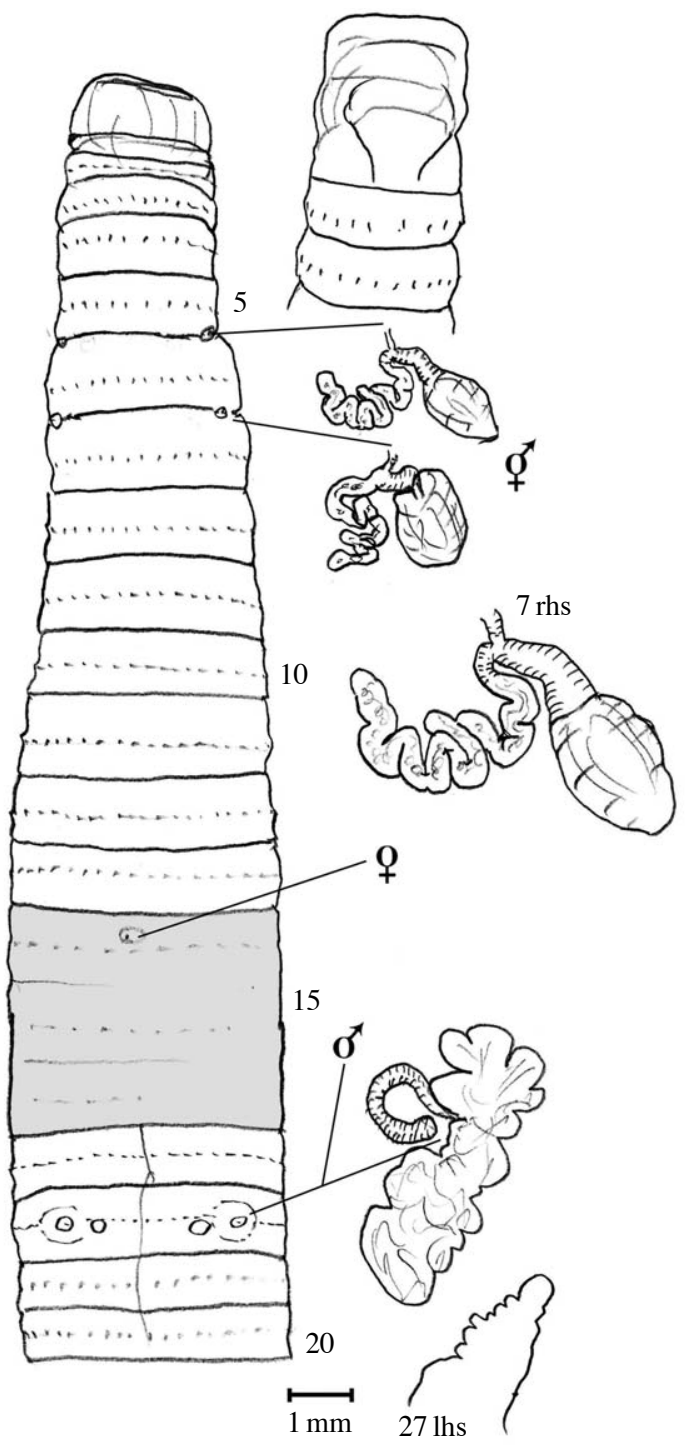

Fig. 8. Amynthas punicans sp. nov. Holotype with $\mathrm{X} 2$ enlargement of 7 rhs spermatheca.

ecal pores in 5/6/7, a character shared with A. fibulus Kobayashi (1936) and A. kobayashii Kobayashi (1938). The first is similar in its serrated caecum but it lacks GMs in 18 and the spermathecae are small and straight as with A. geojeinsulae Song \& Paik (1970); the second has very different male pores and a smooth caecum and can be compared and A. gyeryongensis Hong \& Kim, 2002 that also has short spermathecal diverticula. Other known species in Korea with spermathecae in or near $5 / 6 / 7$ all have male pores in seminal grooves and small diverticula. The DNA data again helps this identity.

16. Amynthas centurio Blakemore sp. nov. (Fig. 9).

Material. IV0000261303 (Holotype DNA sample HY6 


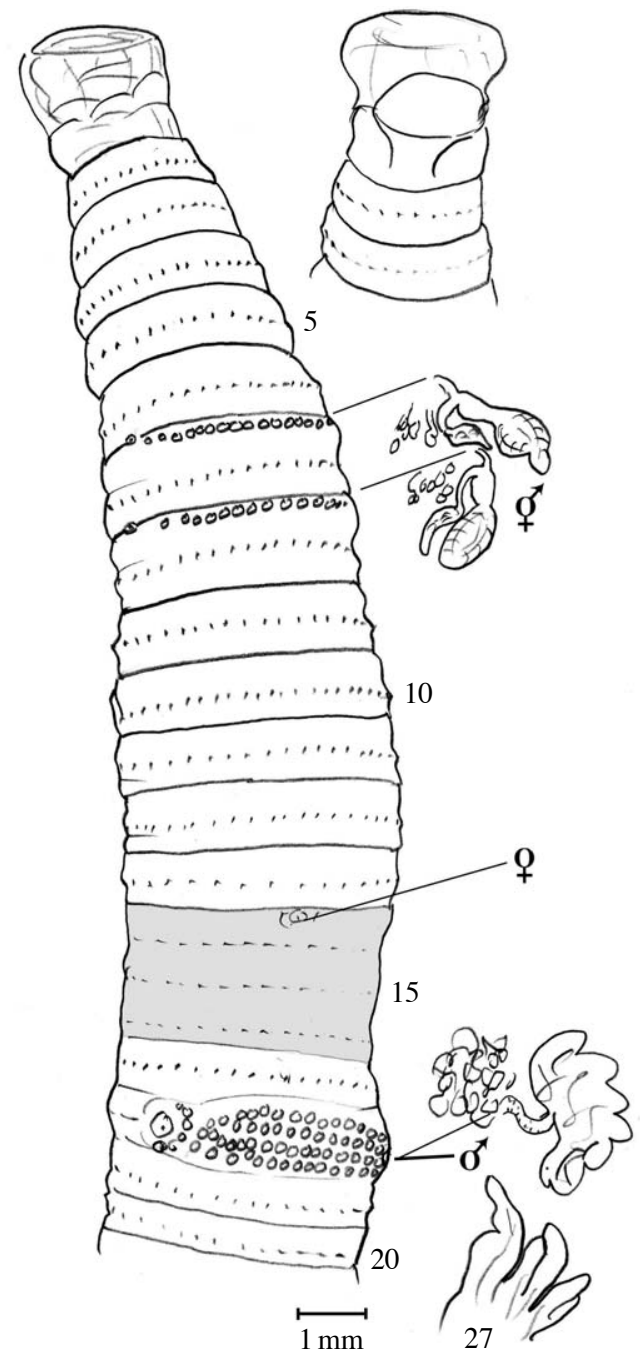

Fig. 9. Amynthas centurio sp. nov. Holotype providing DNA HY6.

- nil results) mature, sketched and dissected from Bangtaesan Mt, $\left(37^{\circ} 55^{\prime} 25.30^{\prime \prime} \mathrm{N} 128^{\circ} 25^{\prime} 48.42^{\prime \prime} \mathrm{E}\right)$, collected by Dr H.-Y. Seo, $11^{\text {th }}$ July, 2013. Sample also contained seven specimens (IV000261304) of three mature M. hilgendorfi and three blue Drawida sp. matures plus an immature. A second container had many specimens but was overcrowded so preservation was poor, all allocated IV000261305.

Etymology. Latin centurio (m noun) for "one hundred" loosely referring to the markings.

Description. Length $65 \mathrm{~mm}$ with 92 segments. Reddish dorsum and pale ventrum, body somewhat transparent. Open epilobous. First dorsal pore 12/13. Perichaetine with ca. 40 setae. Clitellum 14-16 slightly darker. Spermathecae in 6/7/8 laterally ca. 0.5 circumference apart with ca. $18 \mathrm{GM}$ papilla in a row just below intersegmental furrow between each pore. Female pore central on 14. Male pores in setal line on small circular porophore on 18 with a semi-circle of papillae median to each pore and four rows of numerous GM papillae numbering more than sixty. Spermathecal ampullae saccular and slight on short duct with small clavate diverticulum; glands match GMs. Prostates racemose on S-bent muscular duct with glands internally to the GMs. Seminal vesicles large in $11 \& 12$. Caeca manicate in 27.

Remarks. The most distinctive characteristic of the current species from cohorts is the arrangement of GMs numbering about one hundred small papillae in 7/8, 8/9 and especially between the male pores on 18 . The shape of the spermathecae and manicate caeca are also indicative. DNA sequencing was unforthcoming but morphology alone defines this species.

\section{LUMBRICIDAE Rafinesque-Schmaltz, 1815}

\section{Eisenia muuido Blakemore sp. nov. (Fig. 10).}

Material. IV0000261296 (DNA HY30) H, holotype, a mature specimen from Muuido (same details as IV000 261298) collected $15^{\text {th }}$ Sept. 2013 by RJB; IV0000261 297 P, paratype, a subadult lacking tail; plus an immature specimen $(\mathrm{S})$.

Etymology. After island locality (noun in apposition).

Description. Pale body, pinkish in life with distinct yellow anterior and posterior tips (coelomocyctes as in $E$. japonica) found in all life stages $(\mathrm{H}, \mathrm{P}, \mathrm{S})$. Length 100 $\mathrm{mm}(\mathrm{H})$ or $55+\mathrm{mm}(\mathrm{P})$ with 120 segments. Open epilobous. First dorsal pore 4/5. Clitellum weak saddle in ca. 27-32,33. TP elongate bands lateral of b setae on 28-32. Setae ab on 28-32 tumid. Spermathecal pores minute in 9/10/11 dorsally as rounded sacs (one heart-shaped). Female pores in 14 just lateal of $b$ setae. Male pores on barely marked porophores also just lateral of $b$ setae. Nephropores not found. Holandric: testis in seminal vesicles in 9-10 medium sized and larger in 11-12. Hearts in 7-11. Calciferous glands annular in $11 \& 12$. Ovaries in 12 as flattened tongues with many eggs. Intestine from $1 / 215$. Crop in 16. Gizzard in 17-18. Typhlosole not noted. Nephridial bladders sausage-shaped. Much mucous produced when handled.

Remarks. From Michaelsen (1900), the closest agreement is with Eisenia tigrina (Rosa, 1896) from Europe, however it has first dorsal pore in $3 / 4$ and much larger male pores (14/15-15/16). Of the twenty Korean lumbricids listed in Blakemore (2014), the geographically closest are Eisenia japonica (Michaelsen, 1892) and its subspecies (as described by Blakemore \& Grygier, 2011; Blakemore, 2012c; 2013) that have clitella around 24-31 and TP restricted to $27 \& 29$ or E. koreana (Zicsi, 1972), E. gaga Blakemore, 2012, and E. sindo Blakemore, 2012 all three of which differ, however, by having clitella near 25-32 and TP around 27-29. Several of these taxa 


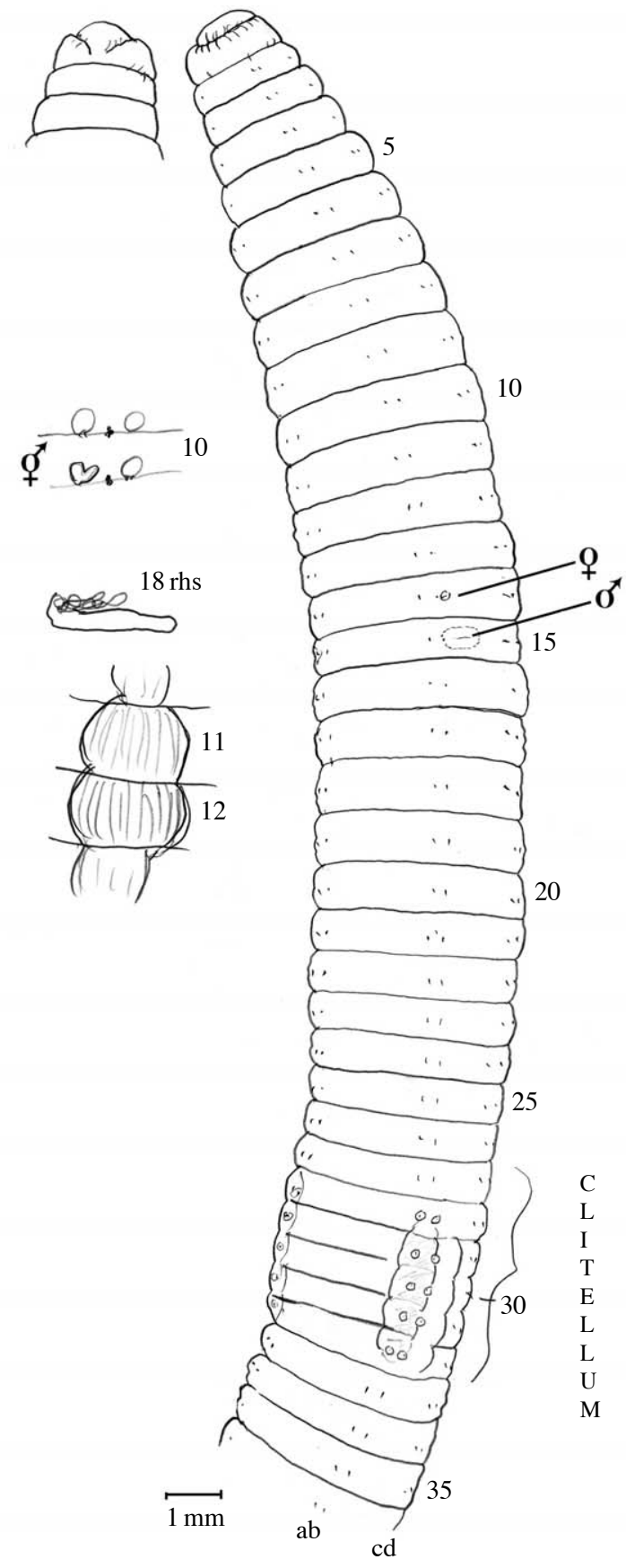

Fig. 10. Eisenia muuido sp. nov. Holotype showing spermathecae, nephridium 18rhs and calciferous glands in 11 \& 12 .

are genetically tested in Appendix and show matches no closer than $79-84 \%$ thus helping to define the uniqueness of the current worm.

DNA barcode Blast results show match no closer than 85-86\% for Eisenia nordenskioldi aff. nordenskioldi (Eisen, 1879) (Genbank JX531498.1 from a Russian study) and its sub-species as redescribed by Blakemore (2013c: tab. 1) that have clitella in the region of 26,27-
32,33 and TP 29-31, thus closest to the current taxon. In Korean Allolobophora species reviewed by Blakemore (2013c: tab. 2), viz. Allolobophora hataii, A. harbinensis and A. dairenensis all by Kobayashi (1940), their clitella are in region of 23,24,25-32,33 and TP in 29-31 only, thus they too differ morphologically.

\section{ACKNOWLEDGements}

The brief Muuido collection trip was self-funded but some others were in part a Korean biodiversity project under auspices of Hanyang University courtesy of Prof. Wonchoel Lee supported by the National Institute of Biological Resources (NIBR) in the Ministry of Environment (MOE) of the Republic of Korea. Sean Prosser and Natalia Ivanova of Canadian Guelph University Centre for Barcoding provided the 'JET' sample DNA results through courtesy of the director Dr Paul Hebert. Thanks for loans of types plus access to library resources are extended to staff at respective museums. Referees and JSR editors kindly made constructive comments yet all taxonomic work in this paper is primary author's.

\section{REFERENCES}

[For brevity, not all taxonomic authorities appended to species binomials are cited here].

Blakemore, R.J. 2000. Tasmanian Earthworms. CD-ROM Monograph with Review of World Families. VermEcology, PO BOX 414 Kippax 2615. Canberra, December, 2000. pp. 800 including 222 figures.

Blakemore, R.J. 2002. Cosmopolitan earthworms - an ecotaxonomic guide to the peregrine species of the World. VermEcology, Kippax, Australia. pp. 506 [CD publication].

Blakemore, R.J. 2003. Japanese earthworms (Annelida: Oligochaeta): a review and checklist of species. Organisms, Diversity and Evolution 3(3):241-244 [Available from: http://www.urbanfischer.de/journals/ode/; www.sencken berg.de/odes/03-11.htm].

Blakemore, R.J. 2006. A series of searchable texts on earthworm biodiversity, ecology and systematics from various regions of the world - Supplemental. Second CD-Rom publication under rules of ICZN (1999). Eds. N. Kaneko \& M. T. Ito. COE Soil Ecology Research Group. Yokohama National University, Japan. - 3rd Edition (2008): http://www.annelida.net/earthworm/ (accessed 13/03/ 2013).

Blakemore, R.J. 2008. Korean earthworm species - updated checklist. In: A series of searchable texts on earthworms - Supplemental. [CD publication compliant with ICZN also provided online as a courtesy from http://www. 
annelida.net/earthworm/Korean.pdf].

Blakemore, R.J. 2010. Saga of Herr Hilgendorf's worms. Zoology in the Middle East 49:7-22.

Blakemore, R.J. 2012a. Amynthas carnosus (Goto and Hatai, 1899) redescribed on its neotype (Oligochaeta : Megadrilacea : Megascolecidae). Journal of Species Research 1(1):35-43 [Available from: www.nibr.go.kr/download? attach_path=journalandattach=2012vol1no1_4.pdf].

Blakemore, R.J. 2012b. On Opening a Box of Worms (Oligochaeta, Megascolecidae) - Historical Earthworm Specimens Transferred to Tokyo from the Saito Ho-on Kai Museum of Natural History in Sendai. - Bulletin of the National Science Museum, Tokyo Series A 38(3):95-124 [Available from: www.kahaku.go.jp/research/.../zoology/ .../BNMNS_A3803_095-124.pdf].

Blakemore, R.J. 2012c. On Schmarda's lost worm and some newly found New Zealand species. Journal of Species Research 1(2):105-132 [Available from: http://www.nibr. go.kr/english/event/journal_spe_3.jsp].

Blakemore, R.J. 2012d. Earthworms from NIBR Jeju-do biosphere compared to historical and new Japanese types (Oligochaeta: Megadrilacea: Megascolecidae). Journal of Species Research 1(2):133-115 [Available from: http:// www.nibr.go.kr/english/event/journal_spe_3.jsp].

Blakemore, R.J. 2012e. Japanese earthworms revisited a decade on (Oligochaeta: Megadrilacea). - Zoology in the Middle East, Supplementum 4:15-22 [Available from: http://www.kasparek-verlag.de/ abstract].

Blakemore, R.J. 2012f. Cosmopolitan earthworms. $5^{\text {th }}$ Edition. VermEcology, Yokohama, Japan. pp. 950 [CD publication].

Blakemore, R.J. 2013a. Jeju-do earthworms (Oligochaeta: Megadrilacea)-Quelpart Island revisited. Journal of Species Research 2(1):15-54 [Available from: www.nibr. go.kr/eng/event/journal_spe_3.jsp].

Blakemore, R.J. 2013b. Ulleung-do earthworms - Dagelet Island revisited. Journal of Species Research 2(1):55-69 [Available from: www.nibr.go.kr/eng/event/journal_spe _3.jsp].

Blakemore, R.J. 2013c. Earthworms newly from Mongolia (Oligochaeta, Lumbricidae, Eisenia). ZooKeys 285:1-21 [Available from: http://www.pensoft.net/journals/ zookeys/article/4502/abstract/earthworms-newly-frommongolia-oligochaeta-, doi: 10.3897/zookeys.285.4502].

Blakemore, R.J. 2013d. Megascolex (Perichaeta) diffringens Baird, 1869 and Pheretima pingi Stephenson, 1925 types compared to the Amynthas corticis (Kinberg, 1867) and A. carnosus (Goto \& Hatai, 1899) species-groups (Oligochaeta: Megadrilacea: Megascolecidae). Journal of Species Research 2(2):99-126 [Available from: http://ocean. kisti.re.kr/downfile/volume/nibr/JOSRB5/2013/v2n2/JO SRB5_2013_v2n2_99.pdf].

Blakemore, R.J. and M.J. Grygier. 2011. Unravelling some Kinki worms (Annelida: Oligochaeta: Megadrili: Lum- bricidae) Part III. Journal of Soil Organisms 83(2):231244 [Available from: www.senckenberg.de/files/content/ ...83_2/06_artikel_blakemore.pdf].

Blakemore, R.J., E. Kupriyanova and M.J. Grygier. 2010. Neotypification of Drawida hattamimizu Hatai, 1930 (Oligochaeta: Megadrili: Moniligastridae) as a model linking mtDNA (COI) sequences to an earthworm type, with a response to the 'Can of Worms' theory of cryptic species. ZooKeys 41:1-29 [Available from: doi: 10.3897/ zookeys.41.374, http://pensoftonline.net/zookeys/index. php/journal/article/view/374/401].

Blakemore, R.J., S. Lee and H.-Y. Seo. 2014. Reports of Drawida (Oligochaeta: Moniligastridae) from far East Asia. Journal of Species Research 3(2):127-166 [Available from: http://www.nibr.go.kr/download?attach_path =journal\&attach=2014vol3no2_3.pdf].

Easton, E.G. 1981. Japanese earthworms: a synopsis of the Megadrile species. - Bulletin of the British Museum (Natural History) Zoology 40(2):33-65 [Available from: http://www.archive.org/stream/bulletinofbritis40zoollon d\#page/n43/mode/2up].

Gates, G.E. 1975. Contributions to a revision of the earthworm family Lumbricidae, XVIII. Eisenia japonica (Michaelsen, 1891). Megadrilogica 2(4):1-3.

Goto, S. and S. Hatai. 1898. New or imperfectly known species of earthworms. No. 1. Annotations Zoologicae Japonensis 2:65-78 [Printed $30^{\text {th }}$ Sept. 1898].

Goto, S. and S. Hatai. 1899. New or imperfectly known species of earthworms. No. 2. Annotations Zoologicae Japonensis 3(1):13-24.

Hebert, P.D.N., A. Cywinska, S.L. Ball and J.R. deWaard. 2003. Biological identifications through DNA bar-codes. Proceedings of the Royal Society, B 270:313-321.

Hong, Y. 2000. Taxonomic review of the family Lumbricidae (Oligochaeta) in Korea. The Korean Journal of Systematic Zoology 16(1):1-13 (Also listed as "Animal Systematics, Evolution and Diversity". Available from: http://www.koreascience.or.kr/article/ArticleFullRecord. jsp?cn=DMBRBT_2000_v16n1_1).

Hong, Y. and S.W. James. 2001. New species of Korean Amynthas Kinberg, 1867 (Oligochaeta, Megascolecidae) with two pairs of spermathecae. Revue suisse de Zoologie 108:65-93.

Hong, Y. and S. James. 2013. Three new earthworm species of the genus Amynthas (Clitellata: Megascolecidae) from Mt. Chiak National Park, Korea. Zootaxa 3646:75-81.

Hong, Y. and T.-H. Kim. 2002. Four new earthworms of the genus Amynthas (Oligochaeta: Megascolecidae) from Korea. Korean Journal of Biological Science 6:195-199.

Kobayashi, S. 1936. Distribution and some external characteristics of Pheretima (Ph.) carnosa (Goto et Hatai) from Korea. Science Report of the Tohoku Imperial University 11(1):115-138.

Kobayashi, S. 1937. Preliminary survey of the earthworms 
of Quelpart Island. Science Report of the Tohoku Imperial University (B) 11(3):333-351.

Kobayashi, S. 1938. Earthworms of Korea I. Science Report of the Tohoku Imperial University 13(2):89-170.

Kobayashi, S. 1941. Earthworms of Korea. II. Science Report of the Tohoku Imperial University (B) 16(2):147-156.

Michaelsen, W. 1900. Das Tierreich 10: Vermes. Friedländer and Sohn, Berlin. pp. 757.

Oishi, M. 1934. Earthworms. Zoological Magazine, Tokyo 46:133-134 [in Japanese].
Sims, R.W. and E.G. Easton. 1972. A numerical revision of the earthworm genus Pheretima auct. (Megascolecidae: Oligochaeta) with the recognition of new genera and an appendix on the earthworms collected by the Royal Society North Borneo Expedition. Biological Journal of the Linnaean Society 4:169-268.

Submitted: May 23, 2013 Revised: February 15, 2015 Accepted: February 25, 2015 


\begin{abstract}
Appendix - mtDNA CO1 barcode gene results with analysis via BLAST and MEGA-6
[JETxx samples are the author's iBOL (www.boldsystems.com) project on Japanese Earthworms at Guelph Uni., Canada; WOyy samples were attempted by a student at Ewha Womens' Uni. but were mixed in their lab (as lamented in Blakemore, 2013a) requiring resampling with prefix "w" at Seoul National Uni.; Hzz and HYzz are Hanyang Uni. data of current material. Arranged chronologically, some provisional/superceded taxa names are retained for reason of cross-reference, other names - of primary types - are definitive (cf. Fig. 1). Most Japan specimens were collected by RJB some with help of TJ Tansy (TJT)].
\end{abstract}

> LK172-173 W8 Metaphire californica Lake Biwa sample collected by RJB (2010/11).

atattggaaccttATATTTTATTCTAGGAATCTGAGCAGGGATAATCGGAGCAGGGATAAGATTACTTATTCGCATCGAACTAAGAC AGCCTGGATCATTCCTTGGAAGAGACCAACTATATAATACAATTGTGACAGCACACGCATTTCTAATAATTTTCTTTCTGGTG ATACCAGTATTTATTGGGGGATTTGGAAACTGACTTCTCCCACTAATGTTAGGAACCCCTGACATAGCGTTCCCTCGACTAA ATAACATAAGATTCTGACTACTGCCACCCTCATTAATTCTACTAGTTAGATCCGCGGCAGTAGAAAAGGGAGCAGGTACAG GATGAACAGTGTACCCTCCACTAGCTAGAAACATAGCACACGCAGGTCCATCAGTAGATCTTGCAATTTTCTCACTACATTT AGCAGGTGCCTCATCAATTTTGGGGGCCATCAATTTCATTACCACTGTGATCAACATGCGATGATCAGGCCTACGCCTAGAG CGAATCCCACTATTCGTATGAGCCGTAGTAATCACTGTAGTACTCCTTCTACTATCACTTCCTGTGCTAGCGGGAGCAATTAC TATATTATTAACGGATCGAAACCTAAATACCTCATTCTTCGACCCTGCAGGTGGGGGAGACCCAATTCTGTATCAACACC

> JET168-11|An-421|Amynthas-soulensis|COI-5P AACACTATATTTTATTTTAGGAATTTGAGCTGGAATAATTGGAGCAGGATTAAGACTACTTATTCGAATTGAACTAAGACAG CCTGGCTCTTTCCTGGGAAGAGATCAACTTTATAATACAATCGTAACTGCCCATGCATTTCTAATAATTTTTTTTCTCGTAAT ACCTGTATTTATTGGGGGATTCGGAAATTGATTATTACCTCTTATACTAGGTGCACCCGATATAGCTTTCCCCCGACTTAATA ATATAAGATTCTGGTTGCTCCCTCCTTCCCTTATTTTATTAGTATCCTCAGCAGCCGTAGAAAAAGGTGCTGGAACAGGATG AACAGTATACCCGCCACTTGCAAGGAATATTGCTCATGCTGGGCCCTCAGTAGACCTGGCAATTTTTTCCCTCCATTTAGCTG GTGCATCATCAATTTTAGGAGCAATTAACTTTATTACCACAGTAATTAATATACGATGGTCTGGGCTACGCTTAGAACGAAT TCCCCTATTTGTATGAGCCGTTGTAATTACTGTAGTTCTCCTGCTCCTATCCTTACCTGTCCTAGCCGGTGCTATTACAATACT ACTAACAGATCGAAACCTAAATACATCATTCTTCG

Blast Metaphire soulensis from Sendai, Japan (Genbank AB542663) 592/595 (99\%).

JET028 (partial) vs. JET168 $=205 / 222(92 \%)$ - possible contamination?

HY5 vs. JET168=577/579(99\%).

> WM15 Jeju1 \#8 Sanbangsan Temple M. californica

AGGTGTTGATACAGAATTGGGTCTCCCCCACCTGCAGGGTCGAAGAATGAGGTATTTAGGTTTCGATCCGTTAATAATATAG TAATTGCTCCCGCTAGCACAGGAAGTGATAGTAGAAGGAGTACTACAGTGATTACTACGGCTCATACGAATAGTGGGATTC GCTCTAGGCGTAGGCCTGATCATCGCATGTTGATCACAGTGGTAATGAAATTGATGGCCCCCAAAATTGATGAGGCACCTGC TAAATGTAGTGAGAAAATTGCAAGATCTACTGATGGACCTGCGTGTGCTATGTTTCTAGCTAGTGGAGGGTACACTGTTCAT CCTGTACCTGCTCCCTTTTCTACTGCCGCGGATCTAACTAGTAGAATTAATGAGGGTGGCAGTAGTCAGAATCTTATGTTATT TAGTCGAGGGAACGCTATGTCAGGGGTTCCTAACATTAGTGGGAGAAGTCAGTTTCCAAATCCCCCAATAAATACTGGTATC ACCAGAAAGAAAATTATTAGAAATGCGTGTGCTGTCACAATTGTATTATATAGTTGGTCTCTTCCAAGGAATGATCCAGGCT GTCTTAGTTCGATGCGAATAAGTAATCTTATCCCTGCTCCGATTATCCCTGCTCAGATTCCTAGAATAAAAT BLAST $100 \%$ M. cal or $98 \%$ M. schmardae from China.

> WO55 - Amynthas sp. Cheyonji - nil result. Redone as w31.

$>$ WO62 - M. soulensis Korea, nil result, redone as w38.

>w38 M. soulensis (redo of WO62). NICEM samples results from BLAST 99\% Ischnura asiatica (contamination of a dragonfly from Japan)! Same as w10 and w12 and w15 and w16 and w18 and w19 and w22 and w23 and w26 and w28 and w35.

$>$ w31 A. gracilis insularum (redo of WO55).

AACCCTATATTTTATTCTAGGAATTTGAGCCGGAATAATTGGAGCTGGAATAAGGCTACTTATTCGAATTGAACTCAGACAG CCGGGATCGTTTCTGGGAAGAGATCAACTATATAATACAATTGTAACAGCTCATGCATTTGTAATAATTTTCTTTCTGGTAAT ACCAGTATTCATTGGTGGATTTGGAAACTGACTACTACCTCTAATGCTGGGTACACCAGACATAGCATTTCCGCGACTTAAT AATATAAGATTTTGACTACTCCCCCCGTCACTTATCTTACTAGTAAGATCCGCGGCCGTTGAAAAGGGGGCGGGAACTGGAT GAACAGTATATCCCCCGCTGGCAAGAAATATTGCACATGCTGGTCCATCAGTAGATCTAGCAATCTTCTCACTACACTTGGC AGGAGCATCATCTATTCTTGGGGCCATTAACTTTATTACAACTGTAATTAATATACGATGATCTGGATTACGGCTAGAACGA ATCCCCCTATTTGTATGGGCCGTAGTAATTACTGTAGTACTTCTACTATTATCTCTACCTGTACTAGCCGGAGCTATTACTAT ATTATTAACAGATCGAAACCTTAACACATCATTCTTTGATCCCGCTGGAGGTGGAGACCCTATTCTATATCAACACCTATTT BLAST 100\% "Amynthas gracilis" GenBank vouchers (AB542484.1 \& 542485.1) specimens were both from Ogasawara Islands, on Chichijima and Hahajima; whereas Identities=623/637 (98\%) for two specimens of Amynthas gracilis (AB542489.1 \& 542491.1) from Kyushu and Okinawa. The conclusion was a new sub-species cf. A. gracilis.

>w63 Amynthas gracilis insularum Cheonji 12 June 2012.

TATATTTTATTCTAGGAATTTGAGCCGGAATAATTGGAGCTGGAATAAGGCTACTTATTCGAATTGAACTCAGACAGCCGGG ATCGTTTCTGGGAAGAGATCAACTATATAATACAATTGTAACAGCTCATGCATTTGTAATAATTTTCTTTCTGGTAATACCAG 
TATTCATTGGTGGATTTGGAAACTGACTACTACCTCTAATGCTGGGTACACCAGACATAGCATTTCCGCGACTTAATAATAT AAGATTTTGACTACTCCCCCCGTCACTTATCTTACTAGTAAGATCCGCGGCCGTTGAAAAGGGGGCGGGAACTGGATGAACA GTATATCCCCCGCTGGCAAGAAATATTGCACATGCTGGTCCATCAGTAGATCTAGCAATCTTCTCACTACACTTGGCAGGAG CATCATCTATTCTTGGGGCCATTAACTTTATTACAACTGTAATTAATATACGATGATCTGGATTACGGCTAGAACGAATCCCC CTATTTGTATGGGCCGTAGTAATTACTGTAGTACTTCTACTATTATCTCTACCTGTACTAGCCGGAGCTATTACTATATTATTA ACAGATCGAAACCTTAACACATCATTCTTTGATCCCGCTGGAGGTGGAGACCCTATTCTATATCAACACCTA megaBLAST result Amynthas gracilis (AB542485.1); 100\% from Japan.

BLAST w63 vs. WO54 Identities $=650 / 650(100 \%)$, i.e., WO54 was mixed.

BLAST w63 vs. w31 Identities $=650 / 650(100 \%)-$ QED thus questioning identification of A. gracilis gracilis as was recognized by Blakemore (2012a: 27).

> HY2 M. ryunome Blakemore, 2012; small (116 mm), prostatic Busan specimen (RJB)

TATACTTTATTTTAGGAATTTGAGCCGGAATAATCGGAGCTGGGATAAGCCTACTTATCCGCATTGAACTAAGTCAACCGGG GTCTTTCCTTGGAAGAGACCAGTTATATAATACGATTGTAACAGCACATGCATTCCTCATAATTTTCTTCTTAGTAATACCAG TATTTATTGGGGGGTTCGGAAACTGGTTGCTACCACTAATACTAGGAACACCAGATATAGCATTCCCCCGACTAAATAACAT AAGATTTTGACTACTCCCCCCTTCCCTAATTCTCCTAGTGAGATCAGCTGCCGTAGAAAAAGGAGCAGGTACAGGTTGAACA GTATACCCACCCCTAGCAAGAAATATAGCACATGCGGGCCCCTCAGTAGATCTTGCAATCTTCTCACTACATTTAGCAGGTG CCTCGTCAATTTTAGGAGCTATTAATTTTATTACCACAGTGATCAATATACGATGGTCAGGACTACGACTAGAACGAATTCC ATTATTTGTTTGAGCAGTAATAATTACTGTAGTACTACTACTATTATCACTCCCTGTACTAGCCGGTGCAATTACTATACTAC TAACAGACCGAAATCTTAACACATCCTTCTTTGATCCAGCTGGTGGTGGAGACCCAATTCTATACCAACACTTATTC megaBlast result: Amynthas incongruus (EF077551 from China), Id. (99\% - misidentified).

BLASTn result: HY2 Metaphire ryunome Busan vs. M. ryunome H from Hikone (Tokyo An-457); results: Id. 653/653 (100\%), i.e. same taxon - its wide distribution now allows concession of possibility to yield to prior records of some as yet unknown species from Asia.

HY2 M. ryunome vs. H3 Amynthas masatakae Busan specimen $(180 \mathrm{~mm})$ no prostates; results: Id. 553/652 (85\%), i.e., different taxa as $15 \%$ dissimilar COI gene.

$>$ HY3|Amynthas-cf-corticis|Dongnae-Gardens-Busan - nil result.

$>$ HY4IAmynthas-bangtaesan

AGCAGGAATGAGACTCCTTATTCGAATCGAGCTAAGACAACCAGGATCTTTCCTAGGTAGTGATCAGCTATATAATACAATT GTTACAGCACATGCATTTCTAATAATTTTTTTTCTTGTAATGCCCGTATTTATTGGGGGATTTGGAAACTGACTTCTACCTCTA ATATTAGGTGCTCCTGATATAGCTTTTCCCCGTCTTAATAACATAAGATTTTGATTACTTCCACCTTCACTAATTTTATTAGTG TCCTCGGCGGCTGTAGAAAAAGGTGCAGGTACTGGATGAACAGTATACCCGCCACTTGCAAGAAATATTGCTCATGCGGGA CCCTCAGTAGACCTAGCAATTTTTTCCCTGCATTTAGCTGGGGCGTCCTCAATTCTAGGTGCAATTAACTTTATTACAACAGT AATTAATATACGATGATCTGGATTACGTCTAGAGCGAATTCCCCTATTTGTATGAGCAGTTGTAATTACCGTAGTTCTTCTAC TACTATCTCTACCCGTATTGGCTGGCGCCATCACAATACTACTAACAGATCGAAATTTAAATACATCATTTTTCGATCCGGCA GGAGGTGGAGACCCAATTCTATATCAACACCTA

Blast result: Megascolecidae sp. (AB607052.1), 86\%

HY4 vs. HY9=610/613 (99\%).

$>$ HY5IAmynthas-cf-chiakensis Mt Bangtaesan (=M. soulensis?)

AGTAATTGGAGCAGGAATAAGACTACTTATTCGAATTGAACTAAGACAGCCGGGCTCTTTCCTGGGAAGAGATCAACTTTAT AATACAATCGTAACTGCCCATGCATTTCTAATAATTTTTTTTCTCGTAATACCTGTATTTATTGGGGGATTCGGAAATTGATT ATTACCTCTTATACTAGGTGCACCCGATATAGCTTTCCCCCGACTTAATAATATAAGATTCTGGTTGCTCCCTCCTTCCCTTAT TTTATTAGTATCCTCAGCAGCCGTAGAAAAAGGTGCTGGAACAGGATGAACAGTATACCCGCCACTTGCAAGGAATATTGC TCATGCTGGGCCCTCAGTAGACCTGGCAATTTTTTCCCTCCATTTAGCTGGTGCATCATCAATTTTAGGAGCAATTAACTTTA TTACCACAGTAATTAATATACGATGGTCTGGGCTACGCTTAGAACGAATTCCCCTATTTGTATGAGCCGTTGTAATTACTGTA GTTCTCCTGCTCCTATCCTTACCTGTCCTAGCCGGTGCTATTACAATACTACTAACAGATCGAAACCTAAATACATCATTCTT CGACCCAGCAGGGGGTGGGGACCCTATTTTATATCAACATTTATTTTG

Blast result: Metaphire soulensis (AB542665.1) 621/623 (99\%).

HY5 vs. > w47 of Metaphire soulensis from Ullungdo as described by Blakemore (2013: 62, fig. 7)=99\%.

HY5 vs. JET168=577/579(99\%), i.e. tolerably the same species.

$>$ HY6lAmynthas sp. Bantaesan - nil result

$>$ HY7IAmynthas-seoraksan - nil result.

$>$ HY8IAmynthas-Jeombangsan1

CTATATTTCATTTTAGGTATTTGAGCTGGAATGGTTGGGGCAGGAATAAGACTACTTATTCGAGTTGAACTAAGACAACCGG GCTCTTTCCTAGGAAGAGATCAACTATATAATACAATTGTAACAGCCCATGCATTCCTAATAATTTTTTTTCTTGTAATACCC GTATTTATTGGGGGATTTGGAAATTGATTACTGCCACTAATGTTGGGTGCACCAGATATAGCTTTCCCACGACTTAATAATAT AAGATTTTGATTGCTACCACCTTCACTCATTTTACTAGTATCTTCGGCAGCCGTAGAAAAGGGTGCCGGAACAGGATGAACA GTATACCCTCCACTAGCAAGAAATATTGCACATGCGGGCCCATCAGTAGATCTAGCAATCTTTTCTCTTCACTTAGCTGGAG CTTCCTCAATTTTAGGAGCAATTAACTTTATCACCACAGTAATTAATATACGGTGATCTGGGTTACGTTTAGAACGAATTCCA CTATTTGTATGGGCAGTTGTAATTACTGTAGTACTCCTTCTACTATCTTTACCAGTTTTAGCCGGTGCTATTACAATGTTACTA ACAGATCGAAACCTAAATACATCATTTTTTGACCCGGCTGGTGGTGGAGA 
Blast result: Metaphire vesiculata (AB542689.1), 88\%.

HY8 vs. AB542558 of “A. tokioensis" from Hachioji, Japan=523/614 (85\%).

$>$ HY9|Amynthas-jeombongsan2

CTATACTTCATTTTAGGTATCTGAGCAGGGATAATTGGAGCAGGAATGAGACTCCTTATTCGAATCGAGCTAAGACAACCAG GATCTTTCCTAGGAAGTGATCAGCTATATAATACAATTGTTACAGCACATGCATTTCTAATAATTTTTTTTCTTGTAATGCCC GTATTTATTGGGGGATTTGGAAACTGACTTCTACCTCTAATATTAGGTGCTCCTGATATAGCTTTTCCCCGTCTTAATAACAT AAGATTTTGATTACTTCCACCTTCACTAATTTTATTAGTGTCCTCGGCGGCTGTAGAAAAAGGTGCAGGTACTGGATGAACA GTATACCCACCACTTGCAAGAAATATTGCTCATGCGGGACCCTCAGTAGACCTAGCAATTTTTTCCCTACATTTAGCTGGGG CGTCCTCAATTCTAGGTGCAATTAACTTTATTACAACAGTAATTAATATACGATGATCTGGATTACGTCTAGAGCGAATTCCC CTATTTGTATGAGCAGTTGTAATTACCGTAGTTCTTCTACTACTATCTCTACCCGTATTGGCTGGCGCCATCACAATACTACT AACAGATCGAAATTTAAATACATCATTTTTCGATCCGGCAGGAGGTGGAGACCCAATTCTATATCAACACCTATTT

Blast result: Metaphire communissima (AB542620.1 from Hyogo-ken, Japan), 88\%.

$>$ HY11IAmynthas-jeombongsan3

TATATTTTATTTTAGGCATTTGAGCTGGGATGGTGGGAGCTGGAATAAGGCTCCTTATTCGAATTGAGCTAAGACAACCAGG GTCTTTCCTAGGAAGTGACCAGCTCTATAATACAATTGTAACAGCTCATGCGTTTCTAATAATTTTTTTTCTTGTAATGCCCG TATTTATTGGGGGATTTGGGAATTGGCTTCTACCTTTAATACTGGGAGCGCCGGATATAGCCTTTCCTCGACTAAATAATATA AGATTTTGATTACTACCTCCCTCACTTATTTTACTAGTATCCTCCGCGGCCGTTGAAAAAGGTGCCGGTACAGGGTGAACAG TATATCCACCACTTGCAAGAAATATTGCACATGCGGGACCATCCGTAGATCTAGCAATTTTTTCCCTTCATTTAGCTGGTGCA TCATCAATCTTGGGGGCAATCAACTTTATTACAACAGTAATTAACATACGATGATCTGGAATGCGCTTAGAGCGCATTCCCC TATTTGTATGGGCTGTAGTGATTACTGTAGTTCTTCTACTTCTCTCTTTACCCGTACTTGCTGGTGCTATCACTATACTATTAA CAGATCGAAATCTAAATACATCATTTTTTGATCCCGCTGGAGGTGGGGACCCTATTTTATATCAACACCTATTT

Blast result: Metaphire communissima (AB542623.1 from Hyogo-ken, Japan), 87\%.

HY11 vs. HY9=568/653 (87\%).

$>$ HY12IAmynthas-jeombongsan4

CTATACTTTATTTTAGGGATCTGAGCAGGAATAATTGGAGCTGGGATAAGACTTCTCATTCGAATTGAACTAAGACAGCCTG GATCCTTTCTAGGAAGTGACCAACTATACAATACAATTGTAACAGCTCATGCATTTTTAATGATTTTTTTTCTTGTTATACCA GTATTTATTGGGGGATTTGGAAACTGATTATTACCCCTCATACTCGGCGCACCTGATATGGCATTTCCACGACTAAATAACA TAAGATTCTGATTACTACCCCCCTCGCTTATCCTACTAGTGTCCTCAGCAGCTGTAGAGAAAGGGGCGGGAACAGGGTGAAC AGTATACCCCCCCCTTGCGAGGAATATTGCACATGCTGGTCCATCAGTAGACCTAGCAATTTTCTCTCTTCACTTAGCGGGG GCATCCTCAATTCTTGGTGCAATCAACTTTATTACAACAGTAATTAATATACGCTGATCGGGCCTACGTTTAGAGCGAATCC CGCTATTCGTGTGGGCAGTTGTAATTACCGTAGTACTATTACTACTATCTCTACCAGTTCTAGCAGGTGCCATTACAATACTG CTTACAGATCGAAATCTAAATACATCATTCTTTGACCCTGCT

Blast result: Amynthas tappensis (AB542548.1 from Osaka, Japan), 87\%

$>$ HY13 null result.

$>$ HY14IAmynthas-cf.-gracilis-gracilis-Jeju1

GAGCAGGAATAAGACTACTTATTCGAATTGAGCTCAGACAGCCGGGATCGTTTCTGGGAAGAGATCAATTATATAATACAA TTGTAACAGCTCATGCATTCGTAATAATTTTCTTTCTAGTAATACCAGTATTCATTGGTGGATTTGGAAACTGACTACTACCT CTAATGCTGGGTACACCAGACATAGCATTTCCGCGGCTTAATAATATAAGATTTTGGCTACTCCCCCCGTCACTTATCTTACT AGTAAGATCCGCGGCCGTTGAAAAGGGGGCGGGAACTGGATGGACAGTATATCCCCCGCTGGCAAGAAATATTGCACATGC TGGTCCATCAGTAGATCTAGCAATCTTCTCACTACACTTAGCAGGGGCATCATCTATTCTTGGGGCCATTAACTTTATTACAA CTGTAATTAATATGCGATGATCTGGATTACGGCTAGAGCGAATCCCCCTATTTGTATGGGCCGTAGTAATTACTGTAGTACTT CTACTATTATCTCTACCTGTACTAGCCGGAGCTATTACTATACTATTAACAGATCGAAACCTTAACACATCATTCTTTGATCC CGCTGGAGGTGGAGACCCTATTCTATATCAACACCTATTT

Blast result: Amynthas gracilis (AB542489.1 \& 542491.1 from Japan), 100\%.

$>$ HY15IAmynthas-cf.-gracilis-Jeju2

CCCTATATTTTATTCTAGGAATTTGAGCCGGAATAATTGGAGCAGGAATAAGACTACTTATTCGAATTGAGCTCAGACAGCC GGGATCGTTTCTGGGAAGAGATCAATTATATAATACAATTGTAACAGCTCATGCATTCGTAATAATTTTCTTTCTAGTAATAC CAGTATTCATTGGTGGATTTGGAAACTGACTACTACCTCTAATGCTGGGTACACCAGACATAGCATTTCCGCGGCTTAATAA TATAAGATTTTGGCTACTCCCCCCGTCACTTATCTTACTAGTAAGATCCGCGGCCGTTGAAAAGGGGGCGGGAACTGGATGG ACAGTATATCCCCCGCTGGCAAGAAATATTGCACATGCTGGTCCATCAGTAGATCTAGCAATCTTCTCACTACACTTAGCAG GGGCATCATCTATTCTTGGGGCCATTAACTTTATTACAACTGTAATTAATATGCGATGATCTGGATTACGGCTAGAGCGAAT CCCCCTATTTGTATGGGCCGTAGTAATTACTGTAGTACTTCTACTATTATCTCTACCTGTACTAGCCGGAGCTATTACTATAC TATTAACAGATCGAAACCTTAACACATCATTCTTTGATCCCGCTGGAGGTGGAGACCCTATTCTATATCAACACCTA Blast result: Amynthas gracilis (AB542491.1), 100\%

> HY16IAmynthas-cf.-gracilis-Jeju3

GGAGCAGGAATAAGACTACTTATTCGAATTGAGCTCAGACAGCCGGGATCGTTTCTGGGAAGAGATCAATTATATAATACA ATTGTAACAGCTCATGCATTCGTAATAATTTTCTTTCTAGTAATACCAGTATTCATTGGTGGATTTGGAAACTGACTACTACC TCTAATGCTGGGTACACCAGACATAGCATTTCCGCGGCTTAATAATATAAGATTTTGGCTACTCCCCCCGTCACTTATCTTAC 
TAGTAAGATCCGCGGCCGTTGAAAAGGGGGCGGGAACTGGATGGACAGTATATCCCCCGCTGGCAAGAAATATTGCACATG CTGGTCCATCAGTAGATCTAGCAATCTTCTCACTACACTTAGCAGGGGCATCATCTATTCTTGGGGCCATTAACTTTATTACA ACTGTAATTAATATGCGATGATCTGGATTACGGCTAGAGCGAATCCCCCTATTTGTATGGGCCGTAGTAATTACTGTAGTAC TTCTACTATTATCTCTACCTGTACTAGCCGGAGCTATTACTATACTATTAACAGATCGAAACCTTAACACATCATTCTTTGAT CCCGCTGGAGGTGGAGACCCTATTCTATATCAACACCTAT

Blast result: Amynthas gracilis (AB542491.1), 100\%

$>$ HY17IAmynthas-californica-Jeju

ATCGGAGCAGGGATAAGATTACTTATTCGCATCGAACTAAGACAGCCTGGATCATTCCTTGGAAGAGACCAACTATATAAT ACAATTGTGACAGCACACGCATTTCTAATAATTTTCTTTCTGGTGATACCAGTATTTATTGGGGGATTTGGAAACTGACTTCT CCCACTAATGTTAGGAACCCCTGACATAGCGTTCCCTCGACTAAATAACATAAGATTCTGACTACTGCCACCCTCATTAATT CTACTAGTTAGATCCGCGGCAGTAGAAAAGGGAGCAGGTACAGGATGAACAGTGTACCCTCCACTAGCTAGAAACATAGCA CACGCAGGTCCATCAGTAGATCTTGCAATTTTCTCACTACATTTAGCAGGTGCCTCATCAATTTTGGGGGCCATCAATTTCAT TACCACTGTGATCAACATGCGATGATCAGGCCTACGCCTAGAGCGAATCCCACTATTCGTATGAGCCGTAGTAATCACTGTA GTACTCCTTCTACTATCACTTCCTGTGCTAGCGGGAGCAATTACTATATTATTAACGGATCGAAACCTAAATACCTCATTCTT CGACCCTGCAGGTGGGGGAGACCCAATTCTGTATCAACACCTATTC

Blast result: Metaphire californica (AY960810.1), 100\%; Metaphire schmardae (EF032610) 98\%.

$>$ HY19|Amynthas-cf.-corticis-Jeju

CTATACTTCATTTTAGGAATTTGAGCCGGAATAATTGGGGCTGGAATAAGTCTTCTTATTCGAATCGAATTAAGACAACCTG GGTCATTCCTGGGAAGTGACCAACTTTATAACACAATTGTAACAGCACATGCATTCTTAATAATTTTTTTTCTAGTAATACCA GTATTTATTGGTGGTTTTGGTAATTGACTATTACCACTTATATTGGGGACTCCAGATATAGCATTTCCACGCCTAAATAATAT AAGATTCTGACTATTACCCCCCTCACTCATCTTACTAGTCTCTTCTGCAGCAGTGGAAAAAGGTGCAGGAACAGGATGAACA GTTTACCCACCACTAGCAAGTAACATTGCGCATGCTGGACCATCAGTAGATCTGGCAATTTTCTCACTACACTTAGCAGGGG CATCATCAATTCTAGGTGCAATCAATTTTATTACTACAGTAATTAATATACGATGATCAGGCCTACGACTAGAACGAATTCC CCTATTTGTATGAGCAGTAGTAATTACCGTAGTTCTACTACTTCTATCATTACCAGTACTAGCCGGTGCTATCACAATACTAT TAACAGATCGAAACCTTAATACATCATTCTTTGATCCAGCAGGAGGGGGAGACCCAATTTTA

Blast result: Amynthas corticis (DQ224190.1), 100\% or Amynthas diffringens (EF077548.1), 100\%, this latter probably a mistake for A. corticis (cf. Blakemore, 2013d).

\begin{abstract}
$>$ HY20lAmynthas-tralfamadore-Jeju TAACAGATCGAAACCTCAATACATCATTCTTTGATCCAGCAGGA

Blast result: Amynthas robustus (AB542534.1), 95\%

HY20 vs WO2 A. tralfamadore Holotype $=619 / 621$ (99\%), i.e., probably same taxon.

HY20 vs w29 A. tralfamadore smaller Jeju specimen (redo of WO53)=618/621 (99\%).

HY20 vs w30 A. tralfamadore pale specimen from Jeju Id=619/621 (99\%).

$>$ HY21-HY29 void.
\end{abstract}

CTATACTTTATTCTAGGAATTTGAGCAGGAATAATTGGTGCAGGAATAAGACTACTTATTCGTATTGAGTTAAGACAACCTG GATCCTTCCTTGGTAGGGACCAGCTATATAATACAATTGTAACAGCACATGCATTTTTAATAATCTTCTTTCTAGTAATACCA GTATTTATTGGTGGATTCGGAAACTGGTTACTACCCCTAATACTTGGAACCCCAGATATGGCGTTCCCTCGATTAAATAACA TAAGATTTTGACTACTCCCGCCATCATTAATTTTATTAGTAAGATCTGCCGCGGTAGAAAAGGGGGCTGGCACAGGTTGAAC AGTTTACCCACCCTTAGCAAGAAATATAGCACATGCCGGTCCTTCAGTAGACCTTGCAATTTTCTCACTTCATCTTGCAGGTG CCTCGTCAATTCTTGGAGCTATTAACTTTATCACAACAGTAATTAACATACGATGATCTGGGTTACGTCTAGAACGAATCCC ACTATTTGTGTGAGCAGTAGTAATCACTGTAGTACTATTACTATTGTCCCTACCTGTACTGGCAGGGGCAATTACCATATTAC

$>$ HY30IEisenia-munido

TCTTTATTTTATCCTAGGAGTTTGGGCCGGTATAGTGGGTGCTGGAATAAGACTCCTCATTCGAATCGAATTAAGACAGCCG GGCGCCTTTCTAGGTAGAGATCAACTATATAATACAATTGTCACAGCTCACGCATTTGTAATAATTTTCTTCTTAGTAATACC CGTATTTATTGGTGGATTTGGAAACTGACTTCTGCCCCTAATACTAGGTGCCCCGGATATAGCCTTTCCTCGTCTAAATAACA TAAGATTCTGACTTCTACCACCCTCCCTGATTCTTCTTGTATCCTCCGCGGCAGTAGAAAAAGGTGCGGGAACAGGATGAAC CGTATACCCCCCTCTTTCTAGAAACCTTGCTCATGCTGGCCCTTCTGTAGATCTAGCCATTTTCTCCCTTCACTTAGCTGGAGC CTCATCTATTCTAGGGGCAATTAACTTTATTACAACCGTTATTAACATACGTTGAGCAGGCATACGTCTTGAACGAATTCCCC TATTCGTATGAGCTGTAATTATTACAGTAGTTCTTCTCCTTCTATCTCTCCCAGTACTTGCAGGTGCAATTACTATGTTACTAA CAGACCGAAACCTTAATACTTCATTCTTTGACCCAGCTGGAGGTGGTGATCCCATCCTATATCAACATCTGTTC

Blast result: Eisenia nordenskioldi aff. nordenskioldi (JX531498.1 Russian study), 85\%.

HY30 vs. > WM1 Eisenia gaga Holotype (INV245509 Fig. 1)=523/644 (81\%).

HY30 vs. $>$ WO25 Eisenia sindo Holotype, H IV0000246435 (Fig. 3) $=524 / 651$ (80\%).

HY30 vs. > JET124-11IAn-466.1IEisenia japonica from Japan=550/656 (84\%).

$>$ JET170-11IAn-417IEisenia japonica Enoshima Japan topotype $=548 / 656(84 \%)$.

HY30 vs. $>$ w18b Jeju \#2 Eisenia japonica (redo of WO45) $=535 / 654(82 \%)$.

HY30 vs. > WM6 NZ Arataki "Eisenia japonica" AMNZ86031=513/650 (79\%).

HY30 vs. $>$ wo65 Mongolian Eisenia nordenskioldi onon Blakemore, 2012 Holotype $=540 / 630(86 \%)$.

> HY31IMetaphire-muиido 
TTATATTTCATTTTAGGTATCTGAGCCGGAATAATTGGAGCCGGAATAAGACTCCTTATTCGAATTGAATTAAGACAGCCTG GATCATTTCTAGGCAGGGACCAATTATATAATACAATTGTAACTGCTCATGCATTTTTAATAATTTTCTTTTTAGTTATACCA GTATTTATTGGGGGATTTGGAAATTGATTACTACCACTTATACTAGGAACCCCAGACATAGCCTTTCCACGACTTAATAATA TAAGATTTTGATTATTACCCCCCTCACTTATTCTCCTAGTATCGTCCGCTGCAGTAGAAAAGGGTGCGGGAACAGGATGAAC AGTATATCCCCCACTAGCAAGAAATATTGCACACGCTGGACCTTCAGTGGACCTTGCAATTTTTTCCCTTCATCTTGCTGGTG CATCCTCAATTCTAGGAGCTATTAATTTTATTACTACTGTAATTAATATACGATGATCTGGGCTTCGCTTAGAGCGAATTCCA CTATTTGTATGAGCAGTAGTAATCACAGTAGTACTCCTACTGCTCTCACTTCCAGTTCTTGCTGGGGCCATTACAATATTATT AACAGACCGAAATCTAAATACATCATTCTTCGATCCAGCGGGAGGGGGGGACCCTATTTTATACCAACACCTATTT Blast result: Amynthas sp. 3(JQ911673.1), 87\%.

> AB542558.1IAmynthas tokioensis ex Hachioji, Japan (Minamiya et al., 2010).

OUTGROUP

ACANTHODRILIDAE (sensu Blakemore, 2000 rather than some Gatesian concept).

$>$ JET157-11|An-480|Microscolex dubius ICOI-5P from Lake Biwa Japan collected by RJB.

AACACTATACTTCATTTTAGGAGTATGAGCAGGGATAATTGGAGCTGGGATAAGGCTATTAATCCGTATTGAATTAAGGCAA CCCGGGGCATTCCTAGGTAGAGATCAACTATATAATACAATTGTTACCGCACATGCATTCTTAATAATTTTTTTCCTGGTAAT ACCAGTATTCATTGGAGGATTTGGAAATTGGCTTCTTCCTCTTATATTGGGGGCCCCTGACATAGCATTTCCACGACTTAATA ATATAAGATTTTGACTGCTACCTCCATCCTTAATTCTATTAATTTCATCAGCTGCTGTAGAAAAGGGGGCTGGGACAGGATG AACTGTATATCCACCCCTTGCAAGAAATATAGCTCATGCGGGACCATCTGTAGACTTAGCAATTTTCTCACTTCATCTTGCAG GAGCCTCGTCCATTTTAGGGGCCATTAACTTTATTACTACTGTTGTTAATATACGATCAACAGGTATACGACTAGAACGAGT ACCCTTATTTGTATGAGCAGTAATTATTACAGTGGTTCTACTACTTCTTTCTTTACCCGTGTTAGCTGGGGCCATTACAATACT ATTAACTGATCGTAACCTAAATACCTCCTTCTTTGACCCTTCAGGTGGTGGAGACCCTATCTTATATCAGCATCTATTC

BLAST 620/621 (99\%) Microscolex dubius isolate WELG2 JN870091 from "South Africa" as above; nBLASTAn-479 vs. An-480 653/658 (99\%). This Japanese specimen of Acanthodrilid Microscolex dubius (Fletcher, 1887) from rice-paddy drains at Lake Biwa is a new record for Far East Asia (Blakemore, 2012e). 